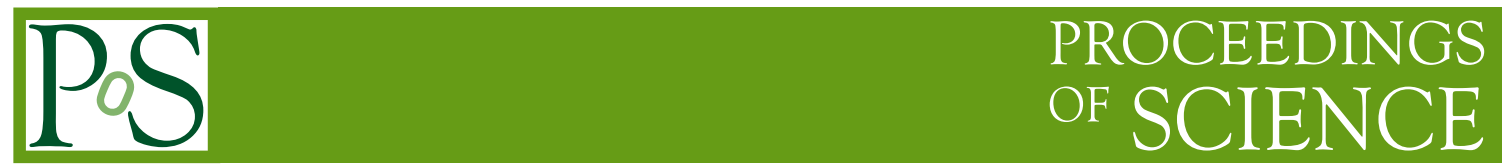

\title{
Why Precision?
}

\section{Johannes Blümlein*}

Deutsches Elektronen-Synchrotron, DESY, Zeuthen, Platanenalle 6, D-15735 Zeuthen, Germany.

Precision measurements together with exact theoretical calculations have led to steady progress in fundamental physics. A brief survey is given on recent developments and current achievements in the field of perturbative precision calculations in the Standard Model of the Elementary Particles and their application in current high energy collider data analyses.

10th International Symposium on Radiative Corrections (Applications of Quantum Field Theory to Phenomenology), RADCOR 2011 Mamallapuram, India September 26-30, 2011

\footnotetext{
* Speaker.
} 


\section{Introduction}

Precision matters. Any progress in the exact sciences relies both on precise measurements and highly accurate theoretical calculations. Many of the fundamental laws of physics had unavoidably to be found whenever precise data were described by theoretical concepts, often within a new framework of relations. The Rudolphine Tables of the late Tycho Brahe [1] led J. Kepler to derive his laws [2] and later I. Newton the law of gravity [3]. A. Michelson's experiments [4] led A. Einstein to Special Relativity [5], with numerous experimental confirmations in flat space-time. ${ }^{1}$ The accurate measurement of the black-body radiation by F. Kurlbaum, H. Rubens, O. Lummer and E. Pringsheim [9] enforced M. Planck to quantize the action [10]. The term measurement of the spectral series by J. Balmer and others [11] led N. Bohr [12] to construct his model of the hydrogen atom. O. Frisch and O. Stern discovered the anomalous magnetic moment of the proton [13]. About 35 years later this phenomenon could be explained by finding the short-distance structure of nucleons as quark and gluon partons by the MIT-SLAC experiments [14]. The discovery of the weak neutral currents by Gargamelle and the polarization-symmetry in deep-inelastic scattering in brilliant experiments clearly indicated the existence of the $W^{ \pm}$and $Z^{0}$ bosons [15], which were discovered by UA1 and UA2 [16] shortly after. M. Veltman [17] found the quadratic mass effects of fermions in the 1-loop electro-weak radiative corrections. The future inclusive electro-weak precision measurements allowed to locate the mass of the top quark, where it was found at Tevatron later [18]. At present, similar more stringent constraints, exploiting the known QCD and electroweak corrections to $e^{+} e^{-}$and $p p$ resp. $\bar{p}$ scattering is setting tighter and tighter mass limits for the Higgs boson [19].

Precision measurements together with precision calculations in the framework of the present Standard Model of the elementary particles and its possible renormalizable extensions allow to search for new phenomena. One may thus expect that the discoveries mentioned before will be followed by various more using precision methods. At the experimental side, key topics are the detailed exploration of the heavy quark sector $(b, t)$ at $B$-factories, the LHC and a future ILC. The masses and mixing parameters in the neutrino sector have to be measured more precisely at $v$ facilities and using astrophysical observations. Another central question concerns the precision measurement of the coupling constants, in particular also of $\alpha_{s}\left(M_{Z}\right)$, which is least known [20]. A major task for the experiments at the LHC consists in the search for the Higgs boson of the Standard Model and possible extensions. If it turns out that the fundamental fermions and bosons do not acquire their masses through the Higgs mechanism, the interaction of the weak bosons will become strong at high energy scales. To investigate this potential phenomenon the LHC experiments need to measure the interaction of weak bosons very precisely. The final task in exploring the new kinematic domain at the LHC is to search for new particles and forces. During this conference two surveys on the present results and the physics potential of ATLAS [21] and CMS [22] were given.

On the side of theoretical computations the level of 4- and 5-loop massless and massive calculations in QCD for zero-scale quantities are performed. $2 \rightarrow n$ scattering processes within the electro-weak theory, QCD, and the MSSM are carried out up to the 2-loop level. Unpolarized and polarized QCD calculations reached the 3-loop level for single differential distributions. The cross

\footnotetext{
${ }^{1}$ At the start of this conference Ref. [6] appeared, which is currently in course of re-analysis [7]. The result has not been confirmed by a more recent measurement [8].
} 
sections for $e p \rightarrow 3$ jets and $p p \rightarrow 2$ jets at next-to-next-to leading order (NNLO) are underway. The predictions for many processes are improved adding appropriate resummations. At the technology side, many calculational tools are developed, partly in close collaboration with mathematical groups. In this way, both the calculations and numerical simulations were greatly improved. In case of various experimental measurements it is presently needed to understand QCD corrections at a level of better than $1 \%$, which requires calculations at the NNLO level and higher. The precise understanding of all 'backgrounds' to the anticipated discoveries is of essential importance and requires all the ongoing theoretical efforts.

\section{Quantum Field Theory}<smiles>[CH]1C=C1</smiles>

\section{Scattering Cross Sections}

\section{介 \\ Mathematics \\ Algorithmics}

The precision measurements at the large scale high-energy facilities like HERA, Tevatron, LHC, the $B$-factories, precision measurements in $v$-physics and planned facilities like the EIC [23] and ILC [24] have driven the theoretical calculations to a much higher level. Many contemporary quantum field theoretic calculations are performed referring to new mathematical methods and are based on an intense use of computer algebra and combinatoric algorithms to end up with precision predictions for scattering cross sections. In this way quantum field theories are understood on the perturbative level a lot better.
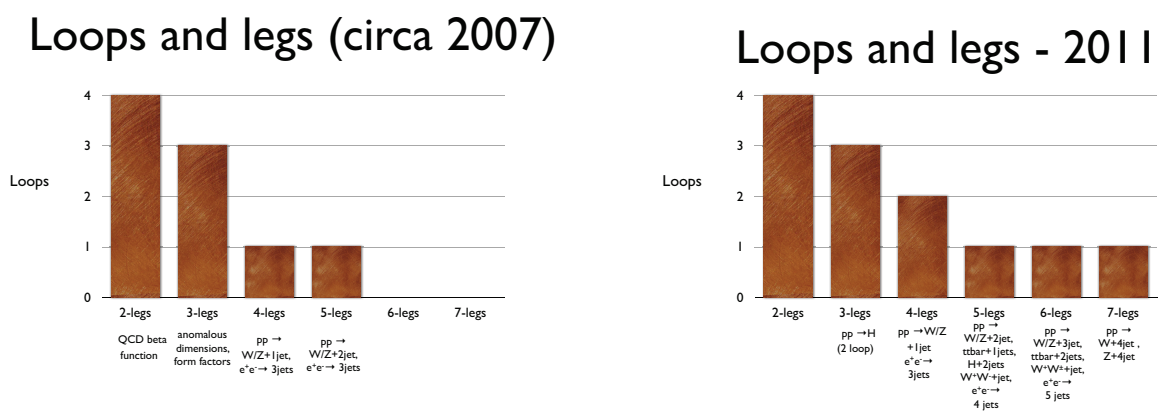

Figure 1: Loops vs. Legs: the development from 2007 to 2011; courtesy by R.K. Ellis [25], reprinted with kind permission.

Keith Ellis has recently summarized the theoretical progress during the last 4-5 years [25], see Fig. 1. This conference adds another entry in column one [26]. 49 contributions where presented on recent results for multi-leg processes at NLO, resummations and infrared structure of scattering cross sections, precision calculations for low-energy processes, multi-loop corrections, mathematical methods for the calculations in quantum field theory, physics at hadron colliders and collider 
phenomenology, and physics beyond the Standard Model. Based on these contributions, I try to give a brief survey on the status of precision calculations currently reached and discuss a few experimental applications.

\section{Multi-Leg Processes}

During the last two years quite a series of important $2 \rightarrow 4(5)$ processes have been calculated at NLO :

$$
\begin{array}{ll}
p p \rightarrow W^{ \pm}(Z, \gamma)+3 \text { jets, } & {[27-32]} \\
p p \rightarrow W^{ \pm}(Z)+4 \text { jets, } & {[33,34]} \\
p p \rightarrow 4 \text { jets, } & {[35]} \\
p p \rightarrow t \bar{t} b \bar{b}, & {[36-38]} \\
p p \rightarrow t \bar{t}+2 \text { jets, } & {[39,40]} \\
p p \rightarrow b \bar{b} b \bar{b}, & {[41,42]} \\
p p \rightarrow t \bar{t} \rightarrow W^{+} W^{-} b \bar{b}, & {[43,44]} \\
p p \rightarrow W^{+} W^{+} 2 \text { jets, } & {[45,46]} \\
p p \rightarrow W^{+} W^{-} 2 \text { jets, } & {[47]} \\
p p \rightarrow W \gamma \gamma+\text { jet, } & {[48]} \\
e^{+} e^{-} \rightarrow \geq 5 \text { jets } & {[49,50]}
\end{array}
$$

These and a series of related processes are of importance for central measurements and searches at the LHC, resp. prepare technical steps in the computation of other processes. In these calculations a series of computational techniques such as MHV amplitudes and their recursion relations, cf. [5154], cutting techniques, cf. [55, 56], and the unitarity method [57] were important, see also [58].

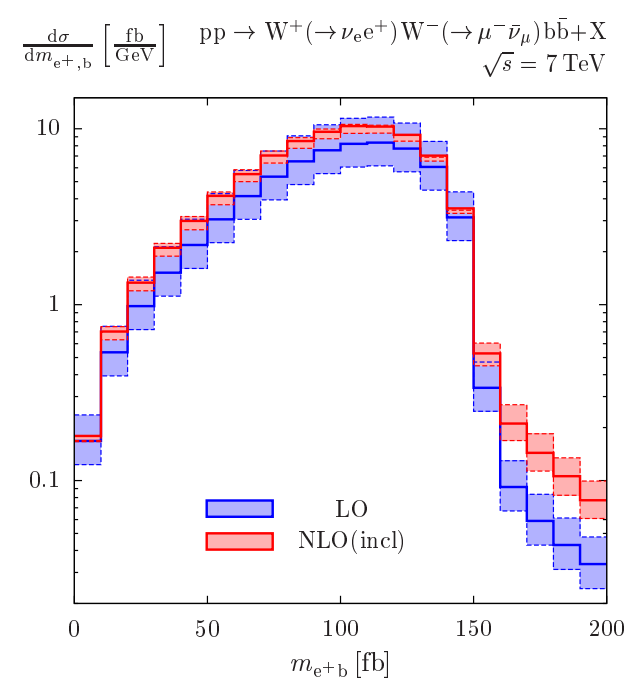

Figure 2: Invariant mass $M_{e^{+} b}$ of the positron- $b$-jet system at the Tevatron: absolute LO and NLO predictions. The uncertainty bands describe $m_{t} / 2<\mu<2 m_{t}$ variations; from A. Denner, S. Dittmaier, S. Kallweit, and S. Pozzorini, Phys. Rev. Lett. 106 (2011) 052001, [44], ${ }^{\odot}(2011)$ by the American Physical Society.

Diagrams contributing to multi-leg processes may contain resonant propagators requiring a special treatment [59]. Furthermore, the calculation of 5-, 6-, 7-point functions may lead to numerical 
instabilities due to large cancellations, which needs special implementations [60].

At this conference reports were given on a variety of multi-leg processes. Hard multi-particle processes at NLO QCD were discussed in [61,62]. The NLO QCD corrections to hadronic $W^{+} W^{-} b \bar{b}$ production have been calculated in $[44,63]$. This process is of importance to measure the $t \bar{t}$ cross section and its background for this channel of final states. As an example we show in Fig. 2 the invariant $M_{e^{+} b}$ mass for the process $p p \rightarrow W^{+} W^{-} b \bar{b}+X$. Results on multi-boson $(+$ jet $)$ production were discussed in $[64,65]$. The tensor reduction algorithm for one-loop multileg Feynman integrals is of importance to obtain stable numerical results. It has been derived and implemented up to seven-point functions in [66]. New results by the GRACE-collaboration have been presented in [67]. The Golem and Samurai projects have recently been united allowing for further advanced calculations [68]. The calculation of the process $W^{+} W^{-} j j$ at NLO was discussed in [69]. A recursive one-loop algorithm for many-particle amplitudes has been presented in [70]. The signal-background interference in $g g \rightarrow H \rightarrow V V$, being not small, has been discussed in [71], see also [72]. Electro-weak and QCD corrections to $p p \rightarrow Z^{*}+$ exponentiation have been implemented in the code Herwiri2. 0 [73]. New aspects in the automation of Standard Model processes in MadLoop were described in [74]. Alternative NLO subtraction schemes were discussed in [75]. Using the Berends-Giele [52,53] and unitarity method, 1-loop corrections to multi jets up to 12-14 gluons were calculated in [76]. At hadron colliders double parton scattering occurs, i.e. two partonic emissions from a single initial state nucleon contribute in a scattering process. For this process electro-weak boson production has been calculated in [77].

Very efficient multi-leg tools at NLO have been created during the last years, which allow the calculation of virtual corrections and real emission. Some of them are equipped with hadronic shower algorithms. A number of packages allow to import newly calculated cross sections for individual reactions in a standardized way. We list a series of codes in alphabetic order.

$\begin{array}{ll}\text { AutoDipole: } & \text { Hasegawa, Moch, Uwer, [78] } \\ \text { BlackHat: } & \text { C. F. Berger, Bern, Dixon, Febres Cordero, Forde, } \\ & \text { Ita, Kosower Maitre, [79] } \\ \text { CutTools: } & \text { Ossola, Papadopoulos, Pittau, [80] } \\ \text { GOLEM: } & \text { Binoth, Guillet, Heinrich, Pilon, Reiter, [81] } \\ \text { GRACE : } & \text { Yuasa, Ishikawa, Kurihara, Fujimoto, Shimizu, } \\ & \text { Hamaguchi, de Doncker, Kato et al., [82] } \\ \text { Helac/Phegas: } & \text { Czakon, Papadopoulos, Worek, [83] } \\ \text { LoopTools: } & \text { Hahn et al. + Feynarts, FormCalc, [84] } \\ \text { MadDipole: } & \text { Frederix, Greiner, Gehrmann, [85] } \\ \text { MadFKS: } & \text { Frederix, Frixions, Maltoni, Stelzer, [86] } \\ \text { MadLoop: } & =\text { CutTools + MadFKS } \\ & \text { Hirschi, Frederix, Frixione, Garzelli, Maltoni, Pittau, [87] } \\ \text { MCFM: } & \text { Campbell, R.K. Ellis, Williams, et al., [88] } \\ \text { MC@NLO: } & \text { Frixione, Webber, [89] } \\ \text { NGluon: } & \text { Badger, Biedermann, Uwer, [90] }\end{array}$




$\begin{array}{ll}\text { NLOJET++: } & \text { Nagy, Trocsanyi, [91] } \\ \text { POWHEG: } & \text { Frixione, Nason, Oleari et al., [92] } \\ \text { Rocket: } & \text { Giele, Zanderighi, [93] } \\ \text { Samurai /GoSam: } & \text { Mastrolia, Ossola, Reiter, Tramontano et al. [94] } \\ \text { SHERPA: } & \text { Gleisberg, Krauss et al., [95] } \\ \text { TeVJet: } & \text { Seymour, Tevlin, [96]. }\end{array}$

There are more packages to be released soon, cf. [97]. We also would like to mention recent implementations of NLO parton showers based on unintegrated kernels [98].

\section{Resummations and Infrared Structure}

For many processes the resummation of large logarithms leads to an improvement of the theoretical description of differential and inclusive scattering cross sections, beyond the available fixed order corrections. In some cases these resummations bridge between the perturbative and nonperturbative range. A general prescription is required to prove the possibility to resum in the particular cases being considered. New insights were provided by soft-collinear effective field theory (SCET). A systematic perturbative approach for resummations beyond the eikonal-approximation has recently been proposed in [99].

Examples for situations in which large logarithms, $L \gg 1$, need to be resummed are [100] :

$$
\begin{aligned}
\text { RGE logs } & \alpha^{k} \ln ^{k}\left(Q^{2} / \mu^{2}\right) \\
\text { High energy logs } & \alpha^{k} \ln ^{k-1}(s / t) \\
\text { Sudakov logs } & \alpha^{k} \ln ^{2 k-1}(1-z), z=\mu_{1}^{2} / \mu_{2}^{2}
\end{aligned}
$$

Coulomb singularity.

Presently resummations are applied for a vast amount of processes: e.g. the large $x$ behaviour of deep-inelastic structure functions, hadronic final states, jet rates, event shapes, the Drell-Yan process, Higgs-boson production, and heavy quark pair production. In the case of Sudakov resummation, the general structure of the scattering cross section in Mellin space reads $[100,101]$

$$
d \sigma\left(\alpha_{s}, N\right)=H\left(\alpha_{s}\right) \exp \left\{\ln (N) g_{1}\left(\alpha_{s}, N\right)+g_{2}\left(\alpha_{s}, N\right) \alpha_{s} g_{3}\left(\alpha_{s}, N\right)+\ldots\right\}+O\left(\frac{1}{N}\right)
$$

and the all-order structure of the perturbative exponent is understood. In other cases, resummations are considered also for transverse momentum spectra.

During this conference a series of contributions were presented on resummations in high energy processes. In [102] the electro-weak gauge-boson production at small $q_{T}$ has been studied at $\mathrm{N}^{3} \mathrm{LL}$. An illustration is given in Fig. 3 comparing to recent data by ATLAS. Using the dipole formula, the soft factor of a generic massless high-energy amplitude has been derived in [103]. This is a promising approach to resum high energy logarithms in a systematic way. The threshold resummation of the total hadronic $t \bar{t}$ cross section at NNLL was carried out in [104, 105], resumming also the Coulomb singularity [104]. The resummation of large $x$ terms in semi-inclusive $e^{+} e^{-}$annihilation was performed in [106]. Amplitude-based resummation in Quantum Field Theories was discussed in [107]. 


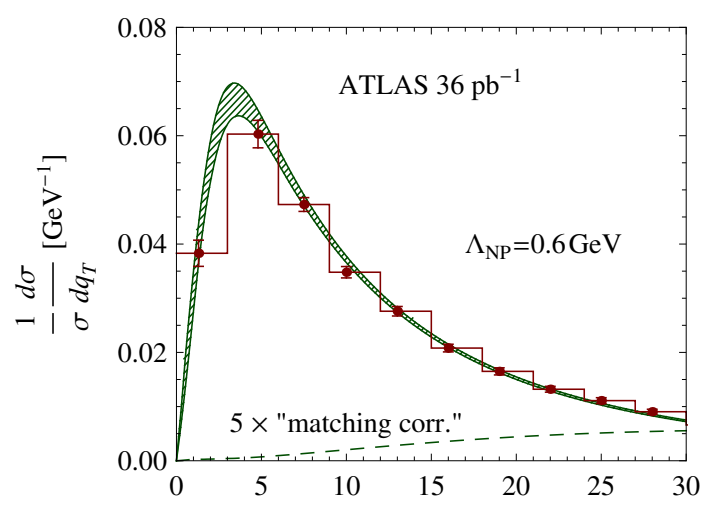

Figure 3: The $q_{T}$-dependence of the resummed Drell-Yan cross section at LHC energies in $\mathrm{N}^{3} \mathrm{LO}$ compared to ATLAS-data; from [102], ${ }^{\odot}(2012)$ Springer Verlag.

\section{Precision Calculations for Low-Energy Processes}

The measurement of fundamental constants and the precise understanding of the associated radiative corrections to the fine structure constant $\alpha_{\mathrm{QED}}\left(m_{e}\right)$ [108], $\left(g_{l}-2\right)$ [109-113], Fermi's constant $G_{F}$ and, related to it, precision physics in atomic systems, cf. e.g. [114], and other pure QED processes, cf. e.g. $[115,116]$, are of great importance. These constants form an important part of the present basis of the Standard Model. Due to the high experimental accuracy being reached, potential effects due to possible extensions of the Standard Model could be revealed comparing with the results of precision calculations. At this conference there were reports on the improvement of $a_{\mu}^{\text {had }}$ taking into account $\rho$-meson width effects [117] and a report on high precision luminosity monitors at low energies [118], as well as an improvement of the $K_{l 3}$ form factor [119].

\section{Multi-Loop Corrections}

Multi-loop calculations in QCD and QED are progressing reaching the 4- resp. 5-loop level and include massive 3-loop results. Various steps forward have been made in case of massless and massive NNLO calculations for $2 \rightarrow 2$ processes in hadronic scattering. All these calculations will allow a much more precise understanding of important Standard Model processes, and related to it, the strong coupling constant, parton distributions, the top-quark mass, jet-physics, and the origin of particle masses, on the basis of upcoming experimental results at the LHC.

At this conference the completed $R_{\text {had }}$-ratio to 4-loops has been reported [120]

$$
\begin{aligned}
R(s)= & 3 \sum_{f} Q_{f}^{2}\left[1+a_{s}+a_{s}^{2}\left(1.986-0.1153 N_{f}\right)+a_{s}^{3}\left(6.637+1.200 N_{f}+0.00518 N_{f}^{2}\right)\right] \\
& -\left(\sum_{f} Q_{f}^{2}\right)^{2}\left[1.2395 a_{s}^{3}-a_{s}^{4}\left(17.8277-0.57489 N_{f}\right)\right]
\end{aligned}
$$


The $\beta$-function of QED is now known analytically to 5-loop order [26] :

$$
\beta_{\mathrm{QED}}=\frac{4}{3} a+4 a^{2}-\frac{62}{9} a^{2}-\left(\frac{5570}{243}+\frac{832}{9} \zeta_{3}\right) a^{4}-\left(\frac{195067}{486}+\frac{800}{3} \zeta_{3}+\frac{416}{3} \zeta_{4}-\frac{6880}{3} \zeta_{5}\right) a^{5} .
$$

Until very recently the gluon-initiated hadronic inclusive production cross section has only been known in the heavy-top approximation. The finite mass effects in the scalar and pseudo-scalar case have been computed in [121,122]. As has been shown in [122] the corrections both in the scalar and pseudo-scalar case are very small for Higgs-masses of $O(120 \mathrm{GeV})$, but amount to $9 \%$ (scalar) resp. $22 \%$ (pseudoscalar) for masses around $m=300 \mathrm{GeV}$. Results of a fully differential NNLO QCD calculations for vector boson and $W$-Higgs production at hadron colliders has been presented in [123] including resummations. The NNLO inclusive Higgs production cross section, including width effects, can be calculated with the code iHixs [124,125]. Comparisons for a wider class of parton distribution functions are provided. In higher order calculations one central problem consists in disentangling of overlapping singularities. One way consists in special non-linear Feynman parameter mapping [126, 127], which allow the numeric calculations of the coefficients in the $\varepsilon$ expansion. The method goes back to Hamberg and van Neerven [128]. The degree of non-linearity of the corresponding representation may limit an eventual analytic calculation, which, however, is not always intended. Non-planar massive double boxes have been calculated based on the code Reduze2 [129]. A related numerical calculation of two-loop box diagrams has been carried out in [67]. Massive Wilson coefficients in the asymptotic region $Q^{2} \gg m^{2}$ factorize into massive operator matrix elements and massless Wilson coefficients [130]. While this has been shown by an explicit calculations to work at $O\left(\alpha^{2}\right)$ in case of massless external lines, a calculation in case of massive on-shell external lines has only been accomplished recently [131] calculating the $O\left(\alpha^{2}\right)$ massive OMEs contributing to the process $e^{+} e^{-} \rightarrow \gamma^{*} / Z^{*}$. Here, the logarithmic terms at $O\left(\alpha^{2}\right)$ yield the desired result, which is not the case for the constant terms, unlike at $O(\alpha)$, needing further investigation. Massive OMEs with massless external lines have been calculated to 3-loops in QCD [132-135] at general values of the Mellin variable $N$ generalizing results obtained for fixed moments in [136], resp. fixed moments in case of two different fermion masses. Currently techniques are available to calculate the bubble- and ladder topologies. The summation methods and codes being used in the calculation have been improved essentially [137].

The knowledge of jet-production cross sections at LHC energies at NNLO is of central importance. This accuracy is required for sensible QCD tests, further constraints on the gluon and sea-quark densities, and a correct background description for various processes. Furthermore, a much deeper insight into QCD-scattering processes is obtained and various valuable new computation technologies are developed for these calculations. The same techniques allow the calculation of a series of other processes like $p p \rightarrow \gamma+$ jet, $2 \gamma, V+$ jet, $V V, H^{0}+$ jet. In earlier works the NNLO corrections to $e^{+} e^{-} \rightarrow 3$ jets $+X$ have been computed, cf. $[138,139]$. Currently the calculation of the NNLO corrections to $p p \rightarrow 2$ jets are underway, with contributions from different groups $[140,141]$. Also the knowledge of the NNLO corrections to $e p \rightarrow 2$ jets $+X$ is of great importance. The available high precision data measured at HERA [142-144] will allow another precision measurement of the $\alpha_{s}\left(M_{Z}^{2}\right)$, still suffering from a large theory-error at NLO. 
Another important process at the LHC is $p p \rightarrow t \bar{t}$. Presently the NNLO corrections are known in approximate form based on NLO + threshold resummation and are available in different codes [145-147]. Recently also the Coulomb corrections have been include [104,148]. The calculation of the complete corrections, forming a challenging task, are presently in progress with contributions from different groups, see [149].

\section{Mathematical Methods in Feynman Diagram Calculations}

Depending on the number of loops, legs, and scales involved in the corresponding problem the calculations can be either performed analytically, semi-analytically, or numerically. Numerical problems are solved using the languages Fortran, $\mathrm{C}$ and $\mathrm{C}++$ at large farms. Many analytic calculations are based on FORM in its versions tform and parform [150], applying multiple threads on main frames or parallelizing to different processors in farms. Other computer algebra codes are written in maple [151], mathemat ica [152], or ginac [153]. Currently typical main frames are equipped with 200-300 Gbyte RAM and fast discs, which are about 10-20 times larger. There are problems for which an amount of 2 peta terms need to be processed [154], which requires run times of the order of one CPU year, to quote an example.

There are general tools for the generation of Feynman diagrams like QGRAF [155] and systematic ways to the Feynman parameter integrals, like graph polynomials [156].

Comparing various calculations the general observation is made that the results have common basis representations, re-appearing in the solution of many different problems [157-159]. In case of 0 -scale problems, such as moments for anomalous dimensions, or expansion coefficients of the $\beta$-function, these are special numbers, like multiple zeta values [154], or corresponding values of iterated integrals at argument $x=1$ [160] over some special alphabets, up to those being generated by cyclotomic polynomials and elliptic integrals, [161-164]. Single scale quantities can be expressed in terms of harmonic sums [165, 166], harmonic polylogarithms [161], hyperlogarithms [167], cyclotomic polylogarithms [163], generalized harmonic sums [168, 169] and other extensions. General structures in case of two- and more scale problems at 2 loops and higher have not been studied systematically yet, but do certainly exist.

Integration and summation methods to solve 0 -scale problems in the massless and massive case have been standardized in several packages like MINCER [170], Baikov's method [171], MATAD [172], qexp [173], a code for 4-loop vacuum-bubble master integrals [174], and Sigma [175]. One may use PSLQ-based [176] methods [154,177,178] to guess the corresponding quantity based on highly precise numerical values, and apply hyperlogarithms ${ }^{2}$ at infinite argument [167].

In the calculation of 1-dimensional and higher dimensional quantities various methods play an important role :

- Integration by parts. Gauß' theorem [179] and relations implied by Lorentz-invariance allow to reduce Feynman integrals to so-called master integrals. There are various implementations of the corresponding algorithms [180], including the recent public codes Air [181], FIRE [182] and REDUZE [129,183].

\footnotetext{
${ }^{2}$ Hyperlogarithms are distinct from the usual iterated integrals with multi-linear denominator functions, since the remainder variables are not constant but may be integrated over.
} 
- Sector decomposition. The decomposition of the integration range of individual Feynman parameter integrals allows the extraction of their singularities [184-187]. Corresponding codes are FIESTA [188], sector_decomposition [189], CSectors [190], SecDec [191].

- Mellin-Barnes integrals. Mellin-Barnes transformations of Feynman parameter integrals [192-194] allow the $\varepsilon$-expansion and the numeric, or in certain cases also the analytic, calculation of the expansion coefficients. Corresponding codes are MB.m, MBasymptotics .m [195], barnesrout ines .m [196], AMBRE . m [197], MBresolve [198].

- Use of differential equations. Master integrals can be often calculated using differential equations [199-201]. There are numerous applications of this method.

- Generalized hypergeometric and related functions. Feynman parameter integrals at 2-loops and in some cases at 3-loops can be represented by these higher transcendental functions $[132,135,202-204]$. Various packages for the expansion in the dimensional parameter $\varepsilon$ around integer and half-integer values exist, as HypExp, HypExp2 and HyperDire [202, 205, 206].

- Difference equations. Feynman diagrams in Mellin space are related by difference equations $[207,208]$. This method was applied systematically e.g. in in [157, 159, 209-212].

- Summation methods. Feynman parameter integrals can be transformed into multiply nested sums over hypergeometric terms. Sums of this kind can be solved in difference- and product fields. Powerful algorithms have been implemented in the package Sigma by C. Schneider [175]. Related to this, the application of multi-sum algorithms may be useful [208,213].

- Recurrences from moments. If a sufficiently large number of moments for a recursive quantity can be generated, one may find its recurrence using the so-called guessing method [214]. It works reliably for very large systems and e.g. allows to reconstruct the 3-loop anomalous dimensions and massless Wilson coefficients. Here up to 5114 moments would be required for individual color factors and the recurrences obtained are of order 35 and degree $\sim 1000$. They can be solved using available summation technologies [159].

- Integration and holonomic functions. In case of holonomic functions the associated multivariate difference or differential equations can in principle be obtained using the AlmkvistZeilberger algorithm [215]. Implementations are given in [216,217].

Relations between harmonic sums $[165,166,218]$ and their generalizations $[168,169]$, resp. harmonic polylogarithms (and generalizations), including cyclotomic harmonic sums, polylogarithms and their generalizations [163] are encoded in packages like summer [165], harmpol [161], hpl [162], nestedsums [219, 220], HPL [221], Xsummer [222], HarmonicSums [217], and CHAPLIN [223]. A large data base for Euler-Zagier values was given in the multiple zeta value data mine [154]. Analytic continuations of harmonic sums to complex arguments $N$ are given in $[163,169,207,224]$. 


\section{Parton Distributions for the LHC}

\subsection{NNLO PDFs}

Let us now discuss some precision measurements in QCD at high energy colliders. The physics at the hadron colliders Tevatron and LHC depends very sensibly on the detailed knowledge of the parton distribution functions (PDFs). During the last decade they were improved steadily. At present they are determined with 3-loop accuracy from the world deep-inelastic and other precise hard scattering data. The five groups $\mathrm{AB}(\mathrm{K}) \mathrm{M}$ [225, 226], HERAPDF [227], JR [228], MSTW08 [229] and NNPDF [230] have carried out NNLO analyses and CTEQ will release NNLO results soon. Precision determinations of PDFs have to refer to consistent precision data. Here the use of the combined H1 and ZEUS data [231] is rather essential. The usual statistical measure $\left(\Delta \chi^{2}=\right.$ $1)$, cf. $[225,226,230]$, should be used, treating the systematic errors separately. Subsamples of precision data should reflect appropriately with their parameters within the global fit. In Fig. 4 the results of present PDF-fits are compared.

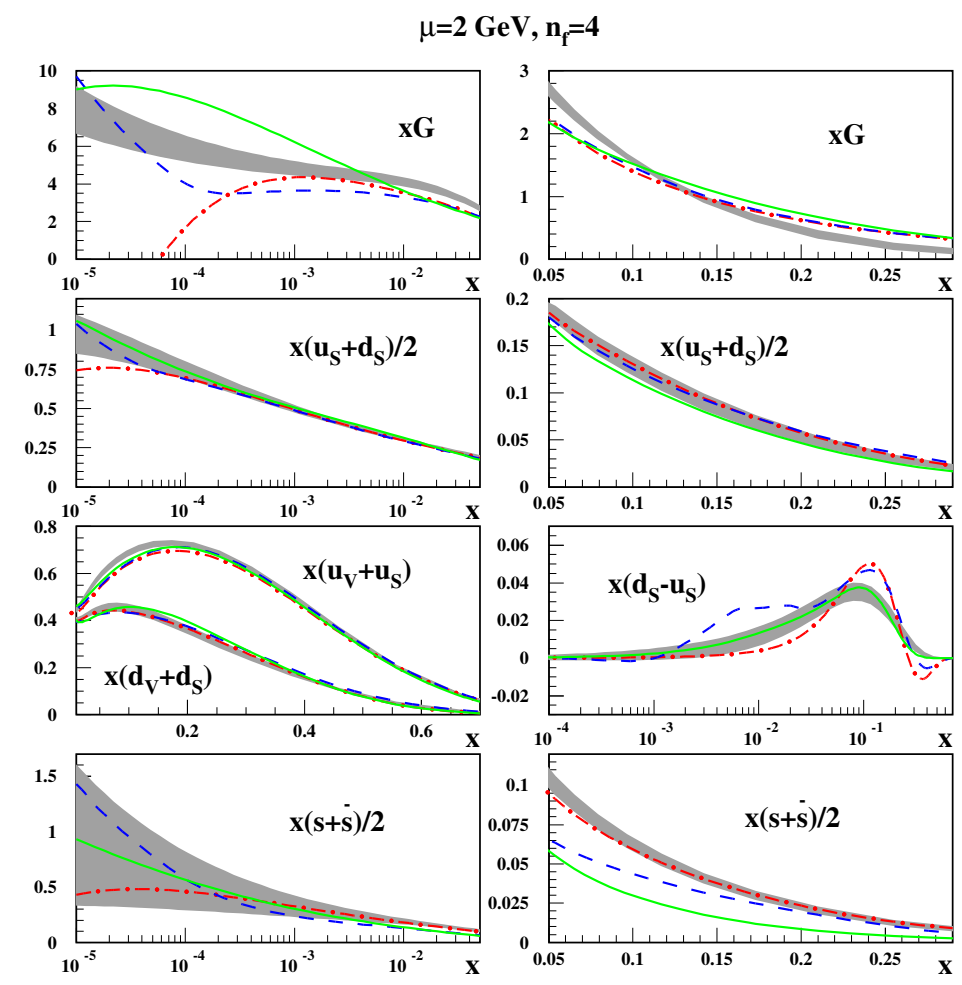

Figure 4: The $1 \sigma$ band for the 4-flavor NNLO ABM11 PDFs [226] at the scale of $\mu=2 \mathrm{GeV}$ versus $x$ (shaded area) compared with the PDFs obtained by other groups. Solid lines: JR09 [228], dashed dots: MSTW08 [229], dashes: NN21 [230]; from [226].

While the valence and sum of the light sea quark distributions agree rather well, there are still big differences in the gluon distribution, the difference of the light sea quark distributions and in case of the strange sea. These differences have an impact on the predictions of different scattering cross 
sections at the LHC. In Ref. [226] a series of reasons for these differences were analyzed and the discussions between the fitting groups on further improvements are ongoing.

$7.2 \alpha_{s}\left(M_{Z}^{2}\right)$

The determination of the strong coupling constant $\alpha_{s}\left(M_{Z}^{2}\right)$ using various precision measurements and perturbative precision calculations resp. lattice simulations has been discussed recently in detail in Ref. [20]. Due to the high accuracy we will compare only the determinations at NNLO and higher, see Table 1.

\begin{tabular}{|l|l|l|}
\hline & \multicolumn{1}{|c|}{$\alpha_{s}\left(M_{Z}\right)$} & \\
\hline BBG & $0.1134+0.0019$ & valence analysis, NNLO [232, 233] \\
BB & $0.1132 \pm 0.0022$ & valence analysis, NNLO [234] \\
GRS & 0.112 & valence analysis, NNLO [235] \\
ABKM & $0.1135 \pm 0.0014$ & HQ: FFNS $n_{f}=3$ [225] \\
ABKM & $0.1129 \pm 0.0014$ & HQ: BSMN-approach [225] \\
JR & $0.1124 \pm 0.0020$ & dynamical approach [228] \\
JR & $0.1158 \pm 0.0035$ & standard fit [228] \\
ABM11 & $0.1134 \pm 0.0011$ & {$[226]$} \\
MSTW & $0.1171 \pm 0.0014$ & {$[236]$} \\
NN21 & $0.1173 \pm 0.0007$ & {$[230]$} \\
CT10 & $0.118 \pm 0.005$ & {$[237]$} \\
\hline Gehrmann et al. & $0.1153 \pm 0.0017 \pm 0.0023$ & $e^{+} e^{-}$thrust [238] \\
Abbate et al. & $0.1135 \pm 0.0011 \pm 0.0006$ & $e^{+} e^{-}$thrust [239] \\
\hline 3 jet rate & $0.1175 \pm 0.0025$ & Dissertori et al. 2009 [240] \\
Z-decay & $0.1189 \pm 0.0026$ & BCK 2008/12 $\left(\mathrm{N}^{3} \mathrm{LO}\right)[120,241]$ \\
$\tau$ decay & $0.1212 \pm 0.0019$ & BCK 2008 [241] \\
$\tau$ decay & $0.1204 \pm 0.0016$ & Pich 2011 [20] \\
$\tau$ decay & $0.1180 \pm 0.0008$ & Beneke, Jamin 2008 [242] \\
\hline lattice & $0.1205 \pm 0.0010$ & PACS-CS 2009 $(2+1$ fl. $)[243]$ \\
lattice & $0.1184 \pm 0.0006$ & HPQCD 2010 [244] \\
lattice & $0.1200 \pm 0.0014$ & ETM 2012 $(2+1+1$ fl.) [245] \\
\hline BBG & $0.1141 \pm 0.0020$ & valence analysis, $\mathrm{N}^{3} \mathrm{LO}\left(^{*}\right)[232]$ \\
BB & $0.1137 \pm 0.0022$ & valence analysis, $\mathrm{N}^{3} \mathrm{LO}\left(^{*}\right)[234]$ \\
\hline world average & $0.1184 \pm 0.0007$ & {$[246](2009)$} \\
& $0.1183 \pm 0.0010$ & {$[20](2011)$} \\
\hline
\end{tabular}

Table 1: Summary of recent NNLO QCD analyses of the DIS world data, supplemented by related measurements using other processes; from [226].

Flavor non-singlet analyses of the DIS world data were performed in [232, 234, 235], with an accuracy of $\Delta \alpha_{s}\left(M_{Z}\right) \simeq 2 \%$. The difference between the value at $\mathrm{N}^{3} \mathrm{LO}^{*}$ and NNLO amounts to $\sim 0.0007$ indicating the size of remaining uncertainty. A difference of $\Delta \alpha_{s}\left(M_{Z}\right)=0.0006$ due to the treatment of the heavy-flavor corrections was observed. These uncertainties signal the typical 
theory errors remaining at the present level of description. The combined flavor non-singlet and singlet analyses [225, 226, 228] obtained quite similar values. The inclusion of Tevatron jet data, cf. [247], although only a NNLO* analysis, alters this values at most to $\alpha_{s}\left(M_{Z}\right)=0.1149 \pm 0.0012$. Re-analyzes have to be performed as soon as the NNLO corrections become available. Low values of $\alpha_{s}\left(M_{Z}\right)$ have also been found in the analysis of thrust in $e^{+} e^{-}$-annihilation in [238,239]. Larger central values of $\alpha_{s}\left(M_{Z}\right)$ at NNLO are reported by MSTW [236] and NN21 [230]. These fits include a much broader set of hadronic scattering data in the analysis. A detailed discussion of sources causing these higher values has been given in Ref. [226]. A (preliminary) central value of $\alpha_{s}\left(M_{Z}\right)$ reported by CT10 [237] is similar to MSTW and NN21 at NNLO, although accompanied by a rather large uncertainty of $\Delta \alpha_{s}\left(M_{Z}\right)=0.0050$. Larger central values for $\alpha_{s}\left(M_{Z}\right)$ are obtained for the 3-jet rate in $e^{+} e^{-}$annihilation [240] at NNLO and for the Z-decay width at $\mathrm{N}^{3} \mathrm{LO}$ [241]. The present $\alpha_{s}\left(M_{Z}\right)$ values at NNLO extracted from $\tau$-decays vary between 0.1212 and 0.1180 $[20,241,242] . \alpha_{s}\left(M_{Z}^{2}\right)$ was also determined in different lattice simulations.

Despite the high precision on $\alpha_{s}\left(M_{Z}^{2}\right)$ reached for different observables there is no consensus yet reached on the value of $\alpha_{s}\left(M_{Z}^{2}\right)$. The systematics between different measurements has still to be further understood and further precision data are needed. Ideal measurements could be performed at the Giga- $Z$ option at a future linear collider.

\section{3 $W^{ \pm}$and $Z$-boson production}

The inclusive $W^{ \pm}$and $Z$-boson production cross sections belong to the standard candles at the LHC, for which NNLO predictions have been calculated, cf. [248]. The cross sections and their
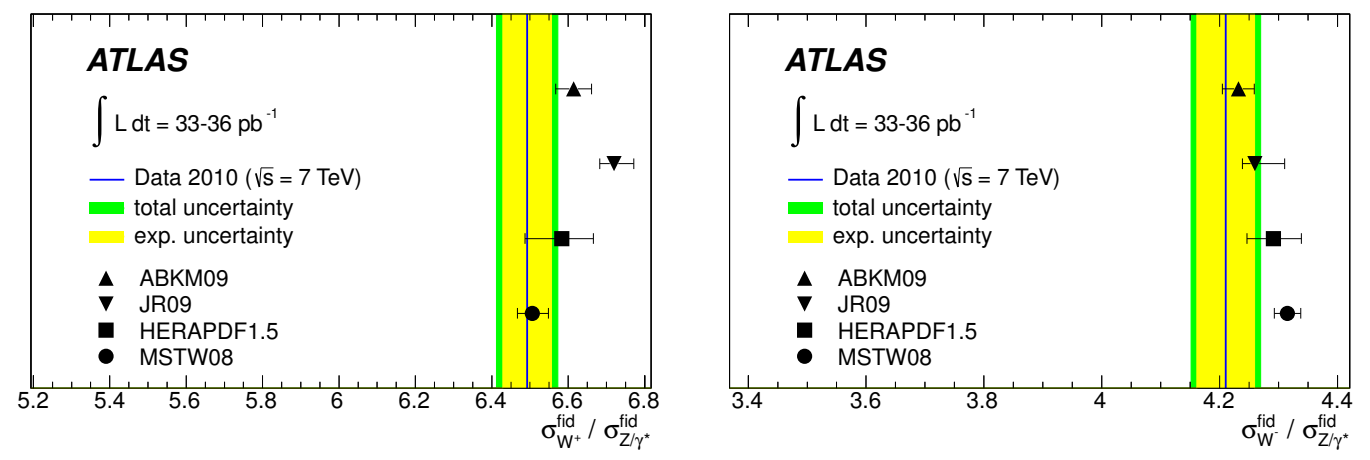

Figure 5: Measured and predicted fiducial cross section ratios, $\sigma\left(W^{+}\right) / \sigma\left(Z^{0}\right)$ (left) and $\sigma\left(W^{-}\right) / \sigma\left(Z^{0}\right)$ (right). The experimental uncertainty (inner yellow band) includes the experimental systematic errors. The total uncertainty (outer green band) includes the statistical uncertainty and the small contribution from the acceptance correction. The uncertainties of the ABKM [225], JR [228] and MSTW08 [229] predictions are given by the PDF uncertainties considered to correspond to $68 \% \mathrm{CL}$ and their correlations are derived from the eigenvector sets. The results for HERAPDF comprise all three sources of uncertainty of that set; from [249], Phys. Rev. D 85 (2012) 072004, ${ }^{\odot}(2012)$ by the American Physical Society.

ratios $\sigma\left(W^{ \pm}\right) / \sigma\left(Z^{0}\right)$ were measured with a high accuracy at ATLAS [249], CMS [250] and $\mathrm{LHCb},[251]$. The cross section ratios are already nearly as precise as the theoretical predictions only taking into account the PDF-errors, see Fig. 5, since the luminosity errors and part of other 
systematic errors cancel. The different predictions do well agree with the measurements and a fit of these data will improve the present accuracy in the sea quark sector.

The LHCb measurements [251] shown in Fig. 6, due to its forward kinematics, are sensitive to the quark and anti-quark distributions at smaller values of $x$. Again, these data will improve the sea-quark distributions.

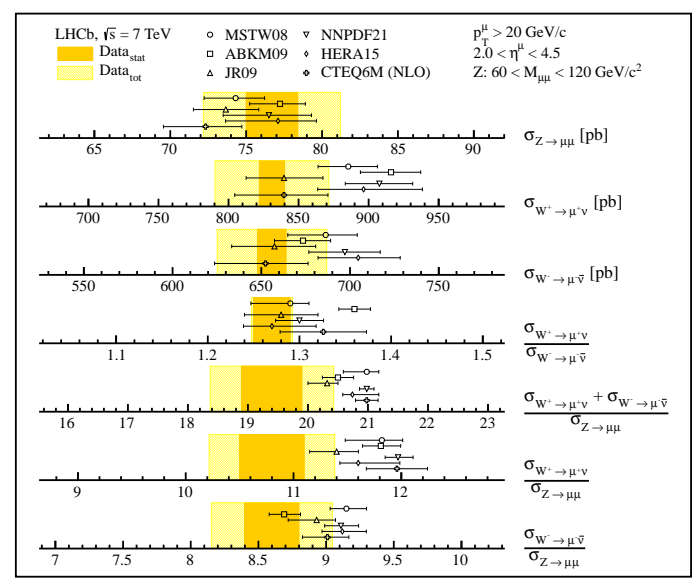

Figure 6: Measurements of the $Z, W^{+}$and $W^{-}$cross-section and ratios, data are shown as bands with the statistical (dark shaded/orange) and total (light hatched/yellow) errors. The measurements are compared to NNLO and NLO predictions with different PDF sets for the proton (ABKM [225], JR [228] and MSTW08 [229], NNPDF [252], and CTEQ [253]), shown as points with error bars. The PDF uncertainty, evaluated at the $68 \%$ confidence level, and the theoretical uncertainties are added in quadrature to obtain the uncertainties of the predictions; from [251] with kind permission of the LHCb collaboration.

\subsection{Jet production}

The jet cross sections at LHC are particularly sensitive to the gluon distribution and the value of $\alpha_{s}\left(M_{Z}^{2}\right)$. Di- and multi-jet final states allow for a better definition of hard scales involved and are expected to allow for more direct comparisons to perturbative predictions. CMS has compared their jet distributions to predictions based on different sets of NLO parton densities [254] in Fig. 7. The dijet mass data show a sensitivity w.r.t. to the different parton distributions at the level of 20 $30 \%$ at NLO. The systematic and scale variation errors are still large. The comparison shows that MSTW [229] and NNPDF [255] give predictions a bit higher than the data, while HERAPDF [227] and ABKM09 [225] are closer to the central values. The data are gluon-dominated and one may expect good constraints from these and other jet data on the gluon distribution in the future.

\subsection{Higgs Search}

The search for the Higgs boson(s) of the Standard Model and of its possible extensions is one of the central tasks of the LHC experiments. The main production process, $g g \rightarrow H^{0}$, depends on the PDFs and $\alpha_{s}\left(M_{Z}^{2}\right)$ like $\alpha_{s}^{2} x G(x) \otimes x G(x)$, where $x G(x)$ denotes the gluon momentum distribution and $\otimes$ the Mellin convolution. The remaining uncertainties both in the PDFs and in $\alpha_{s}\left(M_{Z}^{2}\right)$ propagate into the current prediction for the inclusive Higgs production cross section, cf. e.g. [125], Fig. 8 , which has to be considered in the ongoing experimental analyzes setting exclusion limits 
for Higgs bosons. The current search explores the mass range above $m_{H^{0}}=114.4 \mathrm{GeV}$ [256], with first limits being obtained in [257,258].
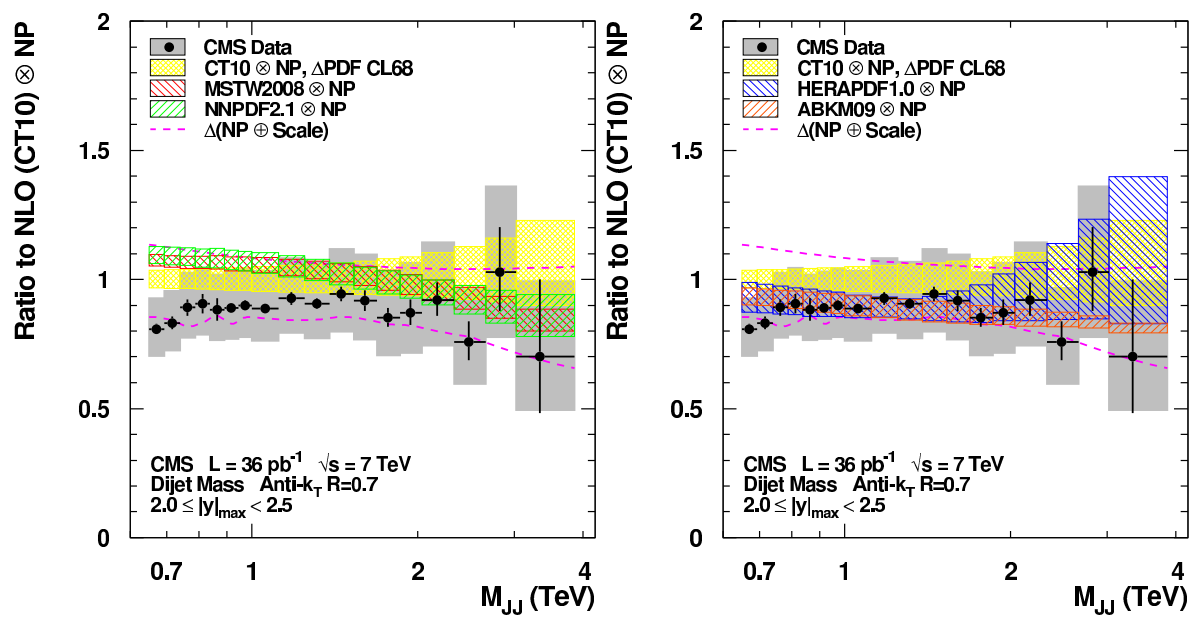

Figure 7: CMS dijet mass data for $2.0<|y|_{\max }<2.5$ are presented vs. $M_{j j}$ with statistical (error bars) as well as systematic uncertainties (grey band) as ratio to NLO using the CT10 PDFs [259]. Additional prediction are shown using the MSTW2008 and NNPDF2.1 (left) and the HERAPDF1.0 [227] and ABKM09 PDFs [225] (right). PDF uncertainties are displayed as colored bands. Common theoretical uncertainties from scale choices and non-perturbative (NP) corrections are indicated by dashed magenta lines; from [254] with kind permission.

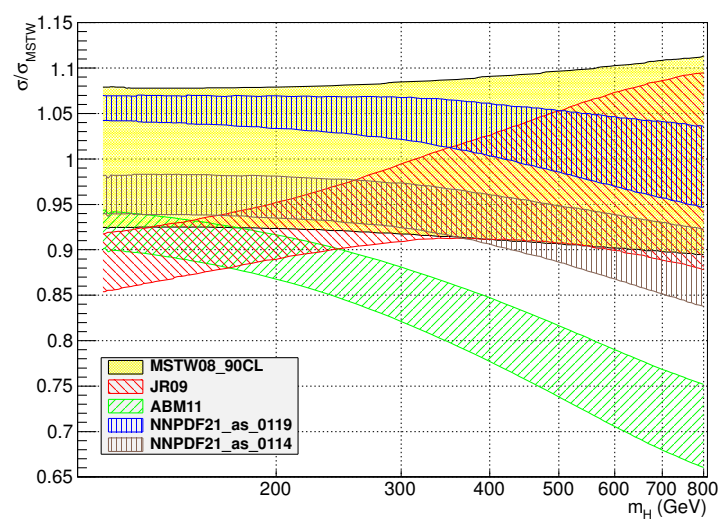

Figure 8: The uncertainty of the Higgs cross-section due to the different sets of parton distribution functions ABM11 [226], JR09 [228], MSTW08 [229], and NN21 [230]; from [125] ${ }^{\circledR}(2012)$ Springer Verlag.

\section{Beyond the Standard Model}

The contributions to the conference which dealt with possible extensions of the Standard Model concerned corrections in the MSSM [260,261] and Kaluza-Klein models [262-264]. There is an increasing number of studies of higher order corrections in renormalizable extensions of the Standard Model, still awaiting experimental evidence. The LHC experiments have carried out 
numerous searches excluding new particles or forces below scales of $\sim 0.5-5 \mathrm{TeV}$, depending on the respective model. Key questions concerning extensions of the Standard Model are :

- Which mechanism generates the masses of the present fundamental particles?

- Do the fundamental forces unify and where?

- Which laws lead to the observed mass spectrum?

- What is the role of gravity?

\section{Summary}

In conclusion, the field of high precision high energy physics is in good shape. Many more precision measurements will be performed at various colliders such as the LHC, lower energy facilities, at JPARC, and at planned facilities like a $B$-factory, the EIC, and ILC. The calculational tools do vastly evolve. The automation of NLO calculations has proceeded very far and includes many important processes already. Highly efficient numerical methods are available. Methods based on computer algebra advance very quickly yielding deeper insight into the analytic structure of quantum field theories. More and more new and longer known mathematical technologies contribute to significant progress in our field. The NLO calculations reached 7-point functions. Resummations are needed in many places to bridge between the perturbative and non-perturbative regions. At NNLO the calculations reached $2 \rightarrow 2$ (3) scattering processes including masses. 4-loop QCD corrections started and more are to come, and first 5-loop results are available. Renormalizable QFTs start to request Tbyte CPUs to solve problems analytically. Sophisticated integrations turn more and more into algebraic problems. Many of the current developments were driven by high precision measurements. Both the precision reached in experiment paired with highly accurate theoretical results lead to a very deep understanding of the structures currently being probed at shortest distances, resp. highest energies. We all enjoy to contribute to and to witness these fascinating and ground-breaking computations in one of the most fundamental fields of science and urgently await to see experimental deviations of these predictions, which are due to new physics.

\section{Acknowledgment.}

I would like to cordially thank the organizers of RADCOR 2011 on behalf of all participants for their excellent work and all their effort to make this conference possible. This work has been supported in part by DFG Sonderforschungsbereich Transregio 9, Computergestützte Theoretische Teilchenphysik, and EU Network LHCPHENOnet PITN-GA-2010-264564.

\section{References}

[1] J. Kepler, Tabulae Rudolphinae, Quibus Astronomicae Scientiae, Temporum longinquitate collapsae Restauratio continetur; A Phoenice illo Astronomorum Tychone, Ex illustri \& Generosa Braheorum in Regno Daniae familia oriundo Equite, (J. Saurii, Ulmae, 1627-[1630]).

[2] J. Kepler, Astronomia nova aitiologetos seu Physica coelestis, (G. Voegelinus, Heidelberg , 1609); J. Kepler, Harmonices Mundi libri V., (G. Tampacchii, Linz , 1619). 
[3] Sir I. Newton, Philosophiae Naturalis Principia Mathematica, (S. Pepys, Londini, 1686); German translation by J. Ph. Wolfers, (R. Oppenheim, Berlin 1872).

[4] A.A. Michelson, American Journal of Science, 22 (1881) 120;

A.A. Michelson and E.W. Morley, American Journal of Science, 34 (1887) 333.

[5] H.A. Lorentz, Proc. Roy. Acad. Sci. Amsterdam 6 (1904) 809;

H. Poincaré, Bull. Sci. Math. 28 (1904) 302;

A. Einstein, Ann. Phys. (Leipzig) 17 (1905) 891.

[6] T. Adam et al. [OPERA Collaboration], arXiv:1109.4897 [hep-ex].

[7] CERN press release Feb. 23 2012, http: / / press . web. cern. ch/press / pressreleases/Releases2011/PR19.11E.html.

[8] M. Antonello et al. [ICARUS Collaboration], arXiv:1203.3433 [hep-ex].

[9] F. Kurlbaum, Ann. Phys. (Leipzig) 65 (1898) 759;

H. Rubens and F. Kurlbaum, Sitz.-Ber. Preuß. Alad. Wiss. (1900) 929;

O. Lummer and E. Pringsheim, Verh. Dtsch. phys. Ges. 2 (1900) 176.

[10] M. Planck, Verh. Dtsch. phys. Ges. 2 (1900) 237.

[11] J.J. Balmer Ann. Physik (Leipzig) 261 (1885) 80;

Th. Lyman Astrophysical J. 23 (1906) 181; Nature 93 (1914) 241;

W. Ritz, Phys. Zeitschr. 11521 (1908) 521;

F. Paschen, Ann. Phys. (Leipzig) 27 (1908) 565.

[12] N. Bohr, Phil. Mag. 26 (1913) 1.

[13] O.R. Frisch and O. Stern, Z. Physik 85 (1933) 4.

[14] E. D. Bloom, D. H. Coward, H. C. DeStaebler, J. Drees, G. Miller, L. W. Mo, R. E. Taylor, M. Breidenbach et al., Phys. Rev. Lett. 23 (1969) 930;

M. Breidenbach, J. I. Friedman, H. W. Kendall, E. D. Bloom, D. H. Coward, H. C. DeStaebler, J. Drees, L. W. Mo et al., Phys. Rev. Lett. 23 (1969) 935.

[15] F. J. Hasert, H. Faissner, W. Krenz, J. Von Krogh, D. Lanske, J. Morfin, K. Schultze, H. Weerts et al., Phys. Lett. B46 (1973) 121;

C. Y. Prescott, W. B. Atwood, R. L. Cottrell, H. C. DeStaebler, E. L. Garwin, A. Gonidec,

R. H. Miller, L. S. Rochester et al., Phys. Lett. B77 (1978) 347.

[16] G. Arnison et al. [ UA1 Collaboration ], Phys. Lett. B122 (1983) 103;

M. Banner et al. [ UA2 Collaboration ], Phys. Lett. B122 (1983) 476.

[17] M. J. G. Veltman, Nucl. Phys. B123 (1977) 89.

[18] F. Abe et al. [ CDF Collaboration ], Phys. Rev. Lett. 74 (1995) 2626 [hep-ex/9503002];

S. Abachi et al. [ D0 Collaboration ], Phys. Rev. Lett. 74 (1995) 2632 [hep-ex/9503003].

[19] Particle data Group, Higgs boson limits.

http://pdg.lbl.gov/2011/figures/figures.html.

[20] S. Bethke, A. H. Hoang, S. Kluth, J. Schieck, I. W. Stewart, S. Aoki, M. Beneke et al., arXiv:1110.0016 [hep-ph].

[21] J. Katzy, ATLAS collaboration, these Proceedings.

[22] M. Pieri, CMS collaboration, these Proceedings. 
[23] E. Aschenauer et al., The EIC Collaboration, A High Luminosity, High Energy Electron-Ion-Collider, A White Paper Prepared for the NSAC LRP 2007;

D. Boer, M. Diehl, R. Milner, R. Venugopalan, W. Vogelsang, D. Kaplan, H. Montgomery and S. Vigdor et al., arXiv:1108.1713 [nucl-th].

[24] J. A. Aguilar-Saavedra et al. [ECFA/DESY LC Physics Working Group Collaboration], hep-ph/0106315;

E. Accomando et al. [ECFA/DESY LC Physics Working Group Collaboration], Phys. Rept. 299 (1998) 1 [hep-ph/9705442].

[25] R.K. Ellis, Summary Talk, “QCD at the LHC”, St. Andrews, Aug., 2011, http : / / conferen ce.ippp.dur.ac.uk/conferenceOtherViews.py?view=ippp\&amp; conf Id=311.

[26] K. Chetyrkin, these Proceedings.

[27] C. F. Berger, Z. Bern, L. J. Dixon, F. Febres Cordero, D. Forde, T. Gleisberg, H. Ita and D. A. Kosower et al., Phys. Rev. D 80 (2009) 074036 [arXiv:0907.1984 [hep-ph]].

[28] C. F. Berger, Z. Bern, L. J. Dixon, F. Febres Cordero, D. Forde, T. Gleisberg, H. Ita and D. A. Kosower et al., Phys. Rev. Lett. 102 (2009) 222001 [arXiv:0902.2760 [hep-ph]].

[29] R. K. Ellis, K. Melnikov and G. Zanderighi, Phys. Rev. D 80 (2009) 094002 [arXiv:0906.1445 [hep-ph]].

[30] K. Melnikov and G. Zanderighi, Phys. Rev. D 81 (2010) 074025 [arXiv:0910.3671 [hep-ph]].

[31] C. F. Berger, Z. Bern, L. J. Dixon, F. Febres Cordero, D. Forde, T. Gleisberg, H. Ita and D. A. Kosower et al., Phys. Rev. D 82 (2010) 074002 [arXiv:1004.1659 [hep-ph]].

[32] J. M. Campbell, R. K. Ellis and C. Williams, Phys. Rev. D 81 (2010) 074023 [arXiv:1001.4495 [hep-ph]].

[33] C. F. Berger, Z. Bern, L. J. Dixon, F. Febres Cordero, D. Forde, T. Gleisberg, H. Ita and D. A. Kosower et al., Phys. Rev. Lett. 106 (2011) 092001 [arXiv:1009.2338 [hep-ph]].

[34] H. Ita, Z. Bern, L. J. Dixon, F. Febres Cordero, D. A. Kosower and D. Maitre, Phys. Rev. D 85 (2012) 031501 [arXiv:1108.2229 [hep-ph]].

[35] Z. Bern, G. Diana, L. J. Dixon, F. Febres Cordero, S. Hoeche, D. A. Kosower, H. Ita and D. Maitre et al., arXiv:1112.3940 [hep-ph].

[36] A. Bredenstein, A. Denner, S. Dittmaier and S. Pozzorini, Phys. Rev. Lett. 103 (2009) 012002 [arXiv:0905.0110 [hep-ph]].

[37] A. Bredenstein, A. Denner, S. Dittmaier and S. Pozzorini, JHEP 1003 (2010) 021 [arXiv:1001.4006 [hep-ph]].

[38] G. Bevilacqua, M. Czakon, C. G. Papadopoulos, R. Pittau and M. Worek, JHEP 0909 (2009) 109 [arXiv:0907.4723 [hep-ph]].

[39] G. Bevilacqua, M. Czakon, C. G. Papadopoulos and M. Worek, Phys. Rev. Lett. 104 (2010) 162002 [arXiv:1002.4009 [hep-ph]].

[40] G. Bevilacqua, M. Czakon, C. G. Papadopoulos and M. Worek, Phys. Rev. D 84 (2011) 114017 [arXiv:1108.2851 [hep-ph]].

[41] T. Binoth, N. Greiner, A. Guffanti, J. Reuter, J. -P. .Guillet and T. Reiter, Phys. Lett. B 685 (2010) 293 [arXiv:0910.4379 [hep-ph]]. 
[42] N. Greiner, A. Guffanti, T. Reiter and J. Reuter, Phys. Rev. Lett. 107 (2011) 102002 [arXiv:1105.3624 [hep-ph]].

[43] G. Bevilacqua, M. Czakon, A. van Hameren, C. G. Papadopoulos and M. Worek, JHEP 1102 (2011) 083 [arXiv:1012.4230 [hep-ph]].

[44] A. Denner, S. Dittmaier, S. Kallweit and S. Pozzorini, Phys. Rev. Lett. 106 (2011) 052001 [arXiv:1012.3975 [hep-ph]].

[45] T. Melia, K. Melnikov, R. Rontsch and G. Zanderighi, JHEP 1012 (2010) 053 [arXiv:1007.5313 [hep-ph]].

[46] N. Greiner, G. Heinrich, P. Mastrolia, G. Ossola, T. Reiter, and F. Tramontano, arXiv:1202.6004.

[47] T. Melia, K. Melnikov, R. Rontsch and G. Zanderighi, Phys. Rev. D 83 (2011) 114043 [arXiv:1104.2327 [hep-ph]].

[48] F. Campanario, C. Englert, M. Rauch and D. Zeppenfeld, Phys. Lett. B 704 (2011) 515 [arXiv:1106.4009 [hep-ph]].

[49] R. Frederix, S. Frixione, K. Melnikov and G. Zanderighi, JHEP 1011 (2010) 050 [arXiv:1008.5313 [hep-ph]].

[50] S. Becker, D. Goetz, C. Reuschle, C. Schwan and S. Weinzierl, Phys. Rev. Lett. 108 (2012) 032005 [arXiv:1111.1733 [hep-ph]].

[51] S. J. Parke and T. R. Taylor, Phys. Rev. Lett. 56 (1986) 2459.

[52] F. A. Berends and W. T. Giele, Nucl. Phys. B 294 (1987) 700.

[53] F. A. Berends and W. T. Giele, Nucl. Phys. B 306 (1988) 759.

[54] M. L. Mangano, S. J. Parke and Z. Xu, Nucl. Phys. B 298 (1988) 653.

[55] E. Witten, Commun. Math. Phys. 252 (2004) 189 [hep-th/0312171];

F. Cachazo, P. Svrcek and E. Witten, JHEP 0409 (2004) 006 [hep-th/0403047];

R. Britto, F. Cachazo and B. Feng, Nucl. Phys. B 715 (2005) 499 [hep-th/0412308];

R. Britto, F. Cachazo, B. Feng and E. Witten, Phys. Rev. Lett. 94 (2005) 181602 [hep-th/0501052].

[56] R. K. Ellis, Z. Kunszt, K. Melnikov and G. Zanderighi, arXiv:1105.4319 [hep-ph].

[57] Z. Bern, L. J. Dixon, D. C. Dunbar and D. A. Kosower, Nucl. Phys. B 425 (1994) 217 [hep-ph/9403226]. Nucl. Phys. B 435 (1995) 59 [hep-ph/9409265].

[58] Z. Bern et al. [NLO Multileg Working Group Collaboration], The NLO multileg working group: Summary report, arXiv:0803.0494 [hep-ph].

[59] See e.g. A. Denner, S. Dittmaier, M. Roth and D. Wackeroth, Nucl. Phys. B 587 (2000) 67 [hep-ph/0006307].

[60] See e.g. J. Fleischer and T. Riemann, Phys. Lett. B 707 (2012) 375 [arXiv:1111.5821 [hep-ph]] and references therein.

[61] A. van Hameren, these Proceedings.

[62] S. Becker, D. Goetz, C. Reuschle, C. Schwan and S. Weinzierl, arXiv:1112.3521 [hep-ph];

[63] S. Pozzorini, these Proceedings.

[64] F. Campanario, C. Englert, M. Rauch, M. Spannowsky and D. Zeppenfeld, arXiv:1112.6336 [hep-ph]. 
[65] P. Agrawal and A. Shivaji, arXiv:1201.0511 [hep-ph].

[66] J. Fleischer, T. Riemann and V. Yundin, arXiv:1202.0730 [hep-ph];

[67] J. Fujimoto, these Proceedings;

F. Yuasa, E. de Doncker, N. Hamaguchi, T. Ishikawa, K. Kato, Y. Kurihara, J. Fujimoto and

Y. Shimizu, arXiv:1112.0637 [hep-ph];

F. Yuasa, T. Ishikawa, Y. Kurihara, J. Fujimoto, Y. Shimizu, N. Hamaguchi, E. de Doncker and

K. Kato, PoS CPP 2010 (2010) 017 [arXiv:1109.4213 [hep-ph]].

[68] G. Cullen, N. Greiner, G. Heinrich, G. Luisoni, P. Mastrolia, G. Ossola, T. Reiter and F. Tramontano, arXiv:1201.2782 [hep-ph].

[69] T. Melia, arXiv:1201.0605 [hep-ph].

[70] Ph. Maierhöfer, these Proceedings.

[71] N. Kauer, arXiv:1201.1667 [hep-ph].

[72] J. M. Campbell, R. K. Ellis and C. Williams, JHEP 1110 (2011) 005 [arXiv:1107.5569 [hep-ph]].

[73] S. Yost, V. Halyo, M. Hejna and B. F. L. Ward, arXiv:1201.5906 [hep-ph].

[74] V. Hirschi, arXiv:1111.2708 [hep-ph].

[75] M. Kubocz, these Proceedings.

[76] S. Badger, B. Biedermann and P. Uwer, arXiv:1201.1187 [hep-ph].

[77] J.R. Gaunt, these Proceedings;

J. R. Gaunt and W. J. Stirling, JHEP 1106 (2011) 048 [arXiv:1103.1888 [hep-ph]].

[78] K. Hasegawa, S. Moch and P. Uwer, Comput. Phys. Commun. 181 (2010) 1802 [arXiv:0911.4371 [hep-ph]];

K. Hasegawa, Eur. Phys. J. C 70 (2010) 285 [arXiv:1007.1585 [hep-ph]].

[79] C. F. Berger, Z. Bern, L. J. Dixon, F. Febres Cordero, D. Forde, H. Ita, D. A. Kosower and D. Maitre, Phys. Rev. D 78 (2008) 036003 [arXiv:0803.4180 [hep-ph]].

[80] G. Ossola, C. G. Papadopoulos and R. Pittau, JHEP 0803 (2008) 042 [arXiv:0711.3596 [hep-ph]].

[81] T. Binoth, J. -P. .Guillet, G. Heinrich, E. Pilon and T. Reiter, Comput. Phys. Commun. 180 (2009) 2317 [arXiv:0810.0992 [hep-ph]].

[82] J. Fujimoto, T. Ishikawa, K. Kato, T. Kaneko, N. Nakazawa, Y. Shimizu, J. Vermaseren and Y. Yasui, Nucl. Phys. Proc. Suppl. 160 (2006) 150;

J. Fujimoto and Y. Kurihara, Nucl. Phys. Proc. Suppl. 183 (2008) 143;

J. Fujimoto, T. Ishikawa, T. Kaneko, K. Kato, S. Kawabata, Y. Kurihara, T. Munehisa and

D. Perret-Gallix et al., Comput. Phys. Commun. 100 (1997) 128 [hep-ph/9605312].

[83] G. Bevilacqua, M. Czakon, M. V. Garzelli, A. van Hameren, A. Kardos, C. G. Papadopoulos,

R. Pittau and M. Worek, arXiv:1110.1499 [hep-ph].

[84] T. Hahn, Nucl. Phys. Proc. Suppl. 89 (2000) 231 [hep-ph/0005029]; Comput. Phys. Commun. 140 (2001) 418 [hep-ph/0012260];

T. Hahn and C. Schappacher, Comput. Phys. Commun. 143 (2002) 54 [hep-ph/0105349];

T. Hahn and M. Rauch, Nucl. Phys. Proc. Suppl. 157 (2006) 236 [hep-ph/0601248].

[85] R. Frederix, T. Gehrmann and N. Greiner, JHEP 1006 (2010) 086 [arXiv:1004.2905 [hep-ph]]. 
[86] R. Frederix, S. Frixione, F. Maltoni and T. Stelzer, JHEP 0910 (2009) 003 [arXiv:0908.4272 [hep-ph]].

[87] V. Hirschi, R. Frederix, S. Frixione, M. V. Garzelli, F. Maltoni and R. Pittau, JHEP 1105 (2011) 044 [arXiv:1103.0621 [hep-ph]].

[88] J. M. Campbell and R. K. Ellis, Phys. Rev. D 60 (1999) 113006 [hep-ph/9905386]; Nucl. Phys. Proc. Suppl. 205-206 (2010) 10 [arXiv:1007.3492 [hep-ph]].

[89] S. Frixione, F. Stoeckli, P. Torrielli, B. R. Webber and C. D. White, arXiv:1010.0819 [hep-ph]; S. Frixione and B. R. Webber, JHEP 0206 (2002) 029 [hep-ph/0204244].

[90] S. Badger, B. Biedermann and P. Uwer, Comput. Phys. Commun. 182 (2011) 1674 [arXiv:1011.2900 [hep-ph]].

[91] Z. Nagy and Z. Trocsanyi, Phys. Rev. D 59, 014020 (1999) [Erratum-ibid. D 62, 099902 (2000)] [hep-ph/9806317]; Phys. Rev. Lett. 87, 082001 (2001) [hep-ph/0104315];

Z. Nagy, Phys. Rev. D 68, 094002 (2003) [hep-ph/0307268];

S. Catani and M. H. Seymour, Nucl. Phys. B 485 (1997) 291 [Erratum-ibid. B 510 (1998) 503] [arXiv:hep-ph/9605323].

[92] S. Frixione, P. Nason and C. Oleari, JHEP 0711 (2007) 070 [arXiv:0709.2092 [hep-ph]].

[93] W. T. Giele and G. Zanderighi, JHEP 0806 (2008) 038 [arXiv:0805.2152 [hep-ph]].

[94] P. Mastrolia, G. Ossola, T. Reiter and F. Tramontano, JHEP 1008 (2010) 080 [arXiv:1006.0710 [hep-ph]].

[95] T. Gleisberg, S. Hoeche, F. Krauss, A. Schalicke, S. Schumann and J. -C. Winter, JHEP 0402 (2004) 056 [hep-ph/0311263].

[96] M. H. Seymour and C. Tevlin, arXiv:0803.2231 [hep-ph].

[97] J. Fleischer, T. Riemann and V. Yundin, DESY-11-252.

[98] M. Skrzypek, S. Jadach, A. Kusina, W. Placzek, M. Slawinska and O. Gituliar, Acta Phys. Polon. B 42 (2011) 2433 [arXiv:1111.5368 [hep-ph]];

M. Slawinska, A. Kusina, S. Jadach and M. Skrzypek, Acta Phys. Polon. B 42 (2011) 1597 [arXiv:1110.0640 [hep-ph]].

[99] E. Laenen, G. Stavenga and C. D. White, JHEP 0903 (2009) 054 [arXiv:0811.2067 [hep-ph]].

[100] For a recent review on resummation in QCD see : L. Magnea, Resummation in QCD, Talk, http://conference.ippp.dur.ac.uk/conferenceotherViews.py?view= ippp\&amp; conf Id=311.

[101] S. Catani, L. Trentadue, G. Turnock and B. R. Webber, Nucl. Phys. B 407 (1993) 3.

[102] T. Becher, M. Neubert and D. Wilhelm, JHEP 1202 (2012) 124 [arXiv:1109.6027 [hep-ph]] and these Proceedings.

[103] V. Del Duca, C. Duhr, E. Gardi, L. Magnea and C. D. White, arXiv:1201.2841 [hep-ph]; JHEP 1112 (2011) 021 [arXiv:1109.3581 [hep-ph]].

[104] M. Beneke, M. Czakon, P. Falgari, A. Mitov and C. Schwinn, Phys. Lett. B 690 (2010) 483 [arXiv:0911.5166 [hep-ph]];

M. Beneke, P. Falgari, S. Klein and C. Schwinn, arXiv:1112.4606 [hep-ph].

[105] A. Hoang and M. Stahlhofen, arXiv:1111.4486 [hep-ph]. 
[106] N. A. Lo Presti, A. Vogt and A. A. Almasy, arXiv:1202.5224 [hep-ph].

[107] B. F. L. Ward, S. K. Majhi and S. A. Yost, arXiv:1201.0515 [hep-ph].

[108] F. Jegerlehner, Nuovo Cim. 034C (2011) 31 [arXiv:1107.4683 [hep-ph]].

[109] K. Melnikov and A. Vainshtein, Theory of the Muon Anomalous Magnetic Moment. (Springer Tracts in Modern Physics, Vol. 216, (Springer, Berlin, 2006).

[110] G. Jegerlehner, The Anomalous Magnetic Moment of the Muon, Springer Tracts in Modern Physics Vol. 226 (Springer, Berlin, 2008).

[111] F. Jegerlehner and A. Nyffeler, Phys. Rept. 477 (2009) 1 [arXiv:0902.3360 [hep-ph]].

[112] T. Aoyama, M. Hayakawa, T. Kinoshita and M. Nio, arXiv:1201.2461 [hep-ph].

[113] A. Czarnecki, X. Garcia i Tormo, and W. J. Marciano, Hyperfine Interactions 210 (2012) 19.

[114] M. Dowling, J. Mondejar, J. H. Piclum and A. Czarnecki, Phys. Rev. A 81 (2010) 022509 [arXiv:0911.4078 [hep-ph]].

[115] A. A. Penin and G. Ryan, JHEP 1111 (2011) 081 [arXiv:1112.2171 [hep-ph]].

[116] C. Carloni Calame, H. Czyz, J. Gluza, M. Gunia, G. Montagna, O. Nicrosini, F. Piccinini and T. Riemann et al., JHEP 1107 (2011) 126 [arXiv:1106.3178 [hep-ph]].

[117] F. Jegerlehner and R. Szafron, Eur. Phys. J. C 71 (2011) 1632 [arXiv:1101.2872 [hep-ph]] and these Proceedings.

[118] J. Gluza, M. Gunia, T. Riemann and M. Worek, arXiv:1201.0968 [hep-ph].

[119] A. Gauhar, these Proceedings.

[120] P. A. Baikov, K. G. Chetyrkin, J. H. Kuhn and J. Rittinger, arXiv:1201.5804 [hep-ph];

[121] R. V. Harlander and K. J. Ozeren, JHEP 0911 (2009) 088 [arXiv:0909.3420 [hep-ph]]; A. Pak, M. Rogal and M. Steinhauser, JHEP 1002 (2010) 025 [arXiv:0911.4662 [hep-ph]].

[122] A. Pak, M. Rogal and M. Steinhauser, JHEP 1109 (2011) 088 [arXiv:1107.3391 [hep-ph]].

[123] G. Ferrera, M. Grazzini and F. Tramontano, Phys. Rev. Lett. 107 (2011) 152003 [arXiv:1107.1164 [hep-ph]] and these Proceedings.

[124] S. Buehler, arXiv:1201.0985 [hep-ph];

[125] C. Anastasiou, S. Buehler, F. Herzog and A. Lazopoulos, JHEP 1204 (2012) 004 [arXiv:1202.3638 [hep-ph]].

[126] F. Herzog, arXiv:1201.0516 [hep-ph].

[127] C. Anastasiou, F. Herzog and A. Lazopoulos, JHEP 1103 (2011) 038 [arXiv:1011.4867 [hep-ph]].

[128] R. Hamberg, Second order gluonic contributions to physical quantities, PhD Thesis, Univ. Leiden (1991).

[129] A. von Manteuffel and C. Studerus, arXiv:1201.4330 [hep-ph] and these Proceedings.

[130] M. Buza, Y. Matiounine, J. Smith, R. Migneron and W. L. van Neerven, Nucl. Phys. B 472 (1996) 611 [hep-ph/9601302].

[131] J. Blümlein, A. De Freitas and W. van Neerven, Nucl. Phys. B 855 (2012) 508 [arXiv:1107.4638 [hep-ph]]; arXiv:1111.6480 [hep-ph]. 
[132] J. Ablinger, J. Blümlein, A. Hasselhuhn, S. Klein, C. Schneider and F. Wißbrock, arXiv:1202.2700 [hep-ph].

[133] J. Ablinger, J. Blümlein, S. Klein, C. Schneider and F. Wißbrock, arXiv:1106.5937 [hep-ph].

[134] J. Ablinger, J. Blümlein, S. Klein, C. Schneider and F. Wißbrock, Nucl. Phys. B 844 (2011) 26 [arXiv:1008.3347 [hep-ph]].

[135] J. Blümlein, A. Hasselhuhn, S. Klein and C. Schneider, arXiv:1205.4184 [hep-ph]; J. Ablinger, J. Blümlein, A. Hasselhuhn, S. Klein, C. Schneider and F. Wißbrock, DESY 12-056.

[136] I. Bierenbaum, J. Blümlein and S. Klein, Nucl. Phys. B 820 (2009) 417 [hep-ph/0904.3563].

[137] J. Blümlein, A. Hasselhuhn and C. Schneider, arXiv:1202.4303 [math-ph].

[138] A. Gehrmann-De Ridder, T. Gehrmann, E. W. N. Glover and G. Heinrich, JHEP 0712 (2007) 094 [arXiv:0711.4711 [hep-ph]]; Phys. Rev. Lett. 100 (2008) 172001 [arXiv:0802.0813 [hep-ph]]; A. Gehrmann-De Ridder, T. Gehrmann and G. Heinrich, Nucl. Phys. B 682 (2004) 265 [hep-ph/0311276];

A. Gehrmann-De Ridder, T. Gehrmann and E. W. N. Glover, Nucl. Phys. B 691 (2004) 195 [hep-ph/0403057]; Phys. Lett. B 612 (2005) 36 [hep-ph/0501291]; Phys. Lett. B 612 (2005) 49 [hep-ph/0502110]; JHEP 0509 (2005) 056 [hep-ph/0505111].

[139] S. Weinzierl, JHEP 0907 (2009) 009 [arXiv:0904.1145 [hep-ph]].

[140] J. Currie, arXiv:1111.4810 [hep-ph].

[141] A. Gehrmann-De Ridder, E. W. N. Glover and J. Pires, JHEP 1202 (2012) 141 [arXiv:1112.3613 [hep-ph]];

T. Gehrmann, E. W. N. Glover, T. Huber, N. Ikizlerli and C. Studerus, JHEP 1011 (2010) 102 [arXiv:1010.4478 [hep-ph]];

J. Pires and E. W. N. Glover, Nucl. Phys. Proc. Suppl. 205-206 (2010) 176 [arXiv:1006.1849 [hep-ph]];

G. Abelof and A. G. -D. Ridder, JHEP 1204 (2012) 076 [arXiv:1112.4736 [hep-ph]];

R. Bonciani, A. Ferroglia, T. Gehrmann, A. Manteuffel and C. Studerus, JHEP 1101 (2011) 102 [arXiv:1011.6661 [hep-ph]];

A. Gehrmann-De Ridder, E. W. N. Glover and J. Pires, JHEP 1202 (2012) 141 [arXiv:1112.3613 [hep-ph]];

A. Gehrmann-De Ridder, M. Ritzmann and P. Skands, Phys. Rev. D 85 (2012) 014013 [arXiv:1108.6172 [hep-ph]];

T. Gehrmann and P. F. Monni, JHEP 1112 (2011) 049 [arXiv:1107.4037 [hep-ph]];

R. Boughezal, K. Melnikov and F. Petriello, Phys. Rev. D 85 (2012) 034025 [arXiv:1111.7041 [hep-ph]];

R. Boughezal, A. Gehrmann-De Ridder and M. Ritzmann, JHEP 1102 (2011) 098 [arXiv:1011.6631 [hep-ph]];

A. Daleo, A. Gehrmann-De Ridder, T. Gehrmann and G. Luisoni, JHEP 1001 (2010) 118 [arXiv:0912.0374 [hep-ph]];

P. Bolzoni, G. Somogyi and Z. Trocsanyi, JHEP 1101 (2011) 059 [arXiv:1011.1909 [hep-ph]].

[142] A. Aktas et al., [H1 Collaboration], Phys. Lett. B 653 (2007) 134 [arXiv:0706.3722 [hep-ex]]; S. Chekanov et al., [ZEUS Collaboration], Phys. Lett. B 547 (2002) 164 [hep-ex/0208037]; Nucl. Phys. B 765 (2007) 1 [hep-ex/0608048];

F. D. Aaron et al. [H1 Collaboration], Eur. Phys. J. C 65 (2010) 363 [arXiv:0904.3870 [hep-ex]]; Eur. Phys. J. C 67 (2010) 1 [arXiv:0911.5678 [hep-ex]]. 
[143] G. Grindhammer, arXiv:1112.0224 [hep-ex].

[144] R. Kogler, arXiv:1112.5117 [hep-ex].

[145] S. Moch and P. Uwer, Phys. Rev. D 78 (2008) 034003 [arXiv:0804.1476 [hep-ph]].

[146] M. Aliev, H. Lacker, U. Langenfeld, S. Moch, P. Uwer and M. Wiedermann, Comput. Phys. Commun. 182 (2011) 1034 [arXiv:1007.1327 [hep-ph]].

[147] M. Czakon and A. Mitov, arXiv:1112.5675 [hep-ph].

[148] M. Beneke, P. Falgari, S. Klein, J. Piclum and C. Schwinn, arXiv:1205.0988 [hep-ph].

[149] C. Anastasiou and S. M. Aybat, Phys. Rev. D 78 (2008) 114006 [arXiv:0809.1355 [hep-ph]]; B. Kniehl, Z. Merebashvili, J. G. Körner and M. Rogal, Phys. Rev. D 78 (2008) 094013 [arXiv:0809.3980 [hep-ph]];

R. Bonciani, A. Ferroglia, T. Gehrmann, D. Maitre and C. Studerus, JHEP 0807 (2008) 129 [arXiv:0806.2301 [hep-ph]];

R. Bonciani, A. Ferroglia, T. Gehrmann and C. Studerus, JHEP 0908 (2009) 067 [arXiv:0906.3671 [hep-ph]];

R. Bonciani, A. Ferroglia, T. Gehrmann, A. Manteuffel and C. Studerus, JHEP 1101 (2011) 102 [arXiv:1011.6661 [hep-ph]];

M. Cacciari, M. Czakon, M. L. Mangano, A. Mitov and P. Nason, Phys. Lett. B 710 (2012) 612 [arXiv:1111.5869 [hep-ph]];

M. Czakon, Nucl. Phys. B 849 (2011) 250 [arXiv:1101.0642 [hep-ph]];

I. Bierenbaum, M. Czakon and A. Mitov, Nucl. Phys. B 856 (2012) 228 [arXiv:1107.4384 [hep-ph]];

S. Alioli, S. -O. Moch and P. Uwer, JHEP 1201 (2012) 137 [arXiv:1110.5251 [hep-ph]];

P. Baernreuther, M. Czakon and A. Mitov, arXiv:1204.5201 [hep-ph];

S. Moch, P. Uwer and A. Vogt, arXiv:1203.6282 [hep-ph];

S. Moch, Hard QCD at Higher Orders, Talk,

https://wiki.bnl.gov/conferences/index.php/DIS-2011.

[150] J. A. M. Vermaseren, math-ph/0010025;

M. Tentyukov and J. A. M. Vermaseren, Comput. Phys. Commun. 181 (2010) 1419

[hep-ph/0702279];

M. Tentyukov, J. A. M. Vermaseren and J. Vollinga, PoS ACAT 2010 (2010) 072 [arXiv:1006.2099 [hep-ph]];

[151] Maple 15, http://www.maplesoft.com/products/maple/.

[152] mathematica 8, http: //www.wolfram.com/mathematica/.

[153] Ginac, http://www.ginac.de/.

[154] J. Blümlein, D. J. Broadhurst and J. A. M. Vermaseren, Comput. Phys. Commun. 181 (2010) 582 [arXiv:0907.2557 [math-ph]].

[155] P. Nogueira, J. Comput. Phys. 105 (1993) 279.

[156] C. Bogner and S. Weinzierl, Int. J. Mod. Phys. A 25 (2010) 2585 [arXiv:1002.3458 [hep-ph]].

[157] J. A. M. Vermaseren, A. Vogt and S. Moch, Nucl. Phys. B 724 (2005) 3 [hep-ph/0504242].

[158] J. Blümlein and V. Ravindran, Nucl. Phys. B 716 (2005) 128 [hep-ph/0501178]; Nucl. Phys. B 749 (2006) 1 [hep-ph/0604019];

J. Blümlein and S. Klein, PoS ACAT (2007) 084 [arXiv:0706.2426 [hep-ph]]. 
[159] J. Blümlein, M. Kauers, S. Klein and C. Schneider, Comput. Phys. Commun. 180 (2009) 2143 [arXiv:0902.4091 [hep-ph]].

[160] H. Poincaré, Sur les groupes d'équations linéaires, Acta Math. 4 (1984) 201;

J.A. Lappo-Danielevsky, Mémoirs sur la Théorie des Systèmes Différentiels Linéaires, (Chelsea, New York, 1953);

K.T. Chen, Ann. of Math. (2) 73 (1961) 110.Ann. Math. 97 (1973) 217.

[161] E. Remiddi and J. A. M. Vermaseren, Int. J. Mod. Phys. A 15 (2000) 725 [hep-ph/9905237].

[162] T. Gehrmann and E. Remiddi, Comput. Phys. Commun. 141 (2001) 296 [hep-ph/0107173]; Comput. Phys. Commun. 144 (2002) 200 [hep-ph/0111255].

[163] J. Ablinger, J. Blümlein and C. Schneider, J. Math. Phys. 52 (2011) 102301, [arXiv:1105.6063 [math-ph]].

[164] S. Laporta, Phys. Lett. B 549 (2002) 115 [hep-ph/0210336]; Int. J. Mod. Phys. A 23 (2008) 5007 [arXiv:0803.1007 [hep-ph]];

D. H. Bailey, J. M. Borwein, D. Broadhurst and M. L. Glasser, arXiv:0801.0891 [hep-th].

[165] J. A. M. Vermaseren, Int. J. Mod. Phys. A 14 (1999) 2037 [hep-ph/9806280];

[166] J. Blümlein and S. Kurth, Phys. Rev. D 60 (1999) 014018 [hep-ph/9810241].

[167] F. Brown, Commun. Math. Phys. 287 (2009) 925 [arXiv:0804.1660 [math.AG]].

[168] S. Moch, P. Uwer and S. Weinzierl, J. Math. Phys. 43 (2002) 3363 [hep-ph/0110083].

[169] J. Ablinger, J. Blümlein, and C. Schneider, in preparation.

[170] S. G. Gorishnii, S. A. Larin, L. R. Surguladze and F. V. Tkachov, Comput. Phys. Commun. 55 (1989) 381;

S. A. Larin, F. V. Tkachov and J. A. M. Vermaseren, NIKHEF-H-91-18.

[171] P. A. Baikov, Phys. Lett. B 385 (1996) 404 [hep-ph/9603267].

[172] M. Steinhauser, Comput. Phys. Commun. 134 (2001) 335 [hep-ph/0009029].

[173] R. Harlander, T. Seidensticker, M. Steinhauser, Phys. Lett. B426 (1998) 125, [hep-ph/9712228]; T. Seidensticker, [hep-ph/9905298].

[174] Y. Schröder and A. Vuorinen, JHEP 0506 (2005) 051 [hep-ph/0503209].

[175] C. Schneider, J. Symbolic Comput. 43 (2008) 611, [arXiv:0808.2543v1]; Ann. Comb. 9 (2005) 75; J. Differ. Equations Appl. 11 (2005) 799; Ann. Comb. 14 (4) (2010), [arXiv:0808.2596]; Proceedings of the Workshop Motives, Quantum Field Theory, and Pseudodifferential Operators, held at the Clay Mathematics Institute, Boston University, June 2-13, 2008, Clay Mathematics Proceedings 12 (2010) 285, Eds. A. Carey, D. Ellwood, S. Paycha, S. Rosenberg; Sém. Lothar. Combin. 56 (2007) 1, Article B56b, Habilitationsschrift JKU Linz (2007) and references therein;

J. Ablinger, J. Blümlein, S. Klein, C. Schneider, Nucl. Phys. Proc. Suppl. 205-206 (2010) 110[arXiv:1006.4797 [math-ph]].

[176] H.R.P. Ferguson and D.H. Bailey, D. H. A Polynomial Time, Numerically Stable Integer Relation Algorithm, RNR Techn. Rept. RNR-91-032, Jul. 14, 199. 
[177] D. J. Broadhurst, hep-th/9612012; arXiv:hep-th/9604128;

J. M. Borwein, D. M. Bradley, D. J. Broadhurst and P. Lisonek, Trans. Am. Math. Soc. 353 (2001) 907 [math/9910045 [math-ca]];

D. H. Bailey and D.J. Broadhurst, Math. Comp. 70 (2001), no. 236, 1719 (electronic), [arxiv: math.NA/9905048];

D. H. Bailey, J. M. Borwein, D. Broadhurst and W. Zudilin, Contemp. Math. 517 (2010) 41 [arXiv:1005.0414 [math-ph]].

[178] R. N. Lee, A. V. Smirnov and V. A. Smirnov, Nucl. Phys. B 856 (2012) 95 [arXiv:1108.0732 [hep-th]].

[179] J. Lagrange Nouvelles recherches sur la nature et la propagation du son, Miscellanea Taurinensis, t. II, 1760-61; Oeuvres t. I, p. 263;

C.F. Gauss, Theoria attractionis corporum sphaeroidicorum ellipticorum homogeneorum methodo novo tractate, Commentationes societas scientiarum Gottingensis recentiores, Vol III, 1813, Werke Bd. V pp. 5-7;

G. Green, Essay on the Mathematical Theory of Electricity and Magnetism, (Nottingham, 1828) [Green Papers, pp. 1-115];

M. Ostrogradski, Mem. Ac. Sci. St. Peters., 6, (1831) 39;

G. 't Hooft and M. J. G. Veltman, Nucl. Phys. B 44 (1972) 189;

K. G. Chetyrkin, A. L. Kataev and F. V. Tkachov, Nucl. Phys. B 174 (1980) 345.

[180] S. Laporta, Int. J. Mod. Phys. A 15 (2000) 5087 [hep-ph/0102033].

[181] C. Anastasiou and A. Lazopoulos, JHEP 0407 (2004) 046 [hep-ph/0404258].

[182] A.V. Smirnov, JHEP 10 (2008) 107, [arXiv:0807.3243].

[183] C. Studerus, Comput. Phys. Commun. 181 (2010) 1293 [arXiv:0912.2546 [physics.comp-ph]].

[184] K. Hepp, Commun. Math. Phys. 2 (1966) 301.

[185] T. Binoth and G. Heinrich, Nucl. Phys. B 585 (2000) 741 [hep-ph/0004013].

[186] Z. Nagy and D. E. Soper, Phys. Rev. D 74 (2006) 093006 [hep-ph/0610028].

[187] C. Anastasiou, S. Beerli and A. Daleo, JHEP 0705 (2007) 071 [hep-ph/0703282].

[188] A. V. Smirnov and M. N. Tentyukov, Comput. Phys. Commun. 180 (2009) 735 [arXiv:0807.4129 [hep-ph]].

[189] C. Bogner and S. Weinzierl, Comput. Phys. Commun. 178 (2008) 596 [arXiv:0709.4092 [hep-ph]].

[190] J. Gluza, K. Kajda, T. Riemann and V. Yundin, PoS ACAT 08 (2008) 124 [arXiv:0902.4830 [hep-ph]].

[191] J. Carter and G. Heinrich, Comput. Phys. Commun. 182 (2011) 1566 [arXiv:1011.5493 [hep-ph]].

[192] E.W. Barnes, Proc. Lond. Math. Soc. (2) 6 (1908) 141; Quart. Journ. Math. 41 (1910) 136; H. Mellin, Math. Ann. 68 (1910) 305.

[193] M. C. Bergere and Y. -M. P. Lam, Commun. Math. Phys. 39 (1974) 1; Berlin preprint FUB HEP $74 / 9$;

M. C. Bergere, C. de Calan and A. P. C. Malbouisson, Commun. Math. Phys. 62 (1978) 137.

[194] N. I. Usyukina, Teor. Mat. Fiz. 22 (1975) 300.

[195] M. Czakon, Comput. Phys. Commun. 175 (2006) 559 [hep-ph/0511200]. 
[196] D. Kosower, barnesroutines.m in: http://projects.hepforge.org/mbtools/

[197] J. Gluza, K. Kajda and T. Riemann, Comput. Phys. Commun. 177 (2007) 879 [arXiv:0704.2423 [hep-ph]].

[198] A. V. Smirnov and V. A. Smirnov, Eur. Phys. J. C 62 (2009) 445 [arXiv:0901.0386 [hep-ph]].

[199] M. Caffo, H. Czyz, S. Laporta and E. Remiddi, Acta Phys. Polon. B 29 (1998) 2627 [hep-th/9807119]; Nuovo Cim. A 111 (1998) 365 [hep-th/9805118].

[200] T. Gehrmann and E. Remiddi, Nucl. Phys. B 580 (2000) 485 [hep-ph/9912329].

[201] M. Caffo, H. Czyz and E. Remiddi, Nucl. Phys. B 634 (2002) 309 [hep-ph/0203256].

[202] M. Y. Kalmykov, JHEP 0604 (2006) 056. [hep-th/0602028]; M. Y. Kalmykov, B. F. L. Ward, S. Yost, JHEP 0702 (2007) 040. [hep-th/0612240]; JHEP 0710 (2007) 048. [arXiv:0707.3654 [hep-th]];

M. Y. Kalmykov, B. A. Kniehl, Nucl. Phys. B809 (2009) 365-405. [arXiv:0807.0567 [hep-th]]. V. V. Bytev, M. Y. .Kalmykov and B. A. Kniehl, Nucl. Phys. B 836 (2010) 129 [arXiv:0904.0214 [hep-th]].

[203] J. Ablinger, I. Bierenbaum, J. Blümlein, A. Hasselhuhn, S. Klein, C. Schneider and F. Wissbrock, Nucl. Phys. Proc. Suppl. 205-206 (2010) 242 [arXiv:1007.0375 [hep-ph]].

[204] V. V. Bytev, M. Y. .Kalmykov and B. A. Kniehl, arXiv:1105.3565 [math-ph].

[205] T. Huber and D. Maitre, Comput. Phys. Commun. 175 (2006) 122 [hep-ph/0507094]; Comput. Phys. Commun. 178 (2008) 755-776. [arXiv:0708.2443 [hep-ph]];

[206] S. Weinzierl, J. Math. Phys. 45 (2004) 2656 [hep-ph/0402131].

[207] J. Blümlein, Comput. Phys. Commun. 180 (2009) 2218 [arXiv:0901.3106 [hep-ph]].

[208] J. Blümlein, S. Klein, C. Schneider and F. Stan, J. Symb. Comp. in print, [arXiv:1011.2656 [cs.SC]].

[209] S. Moch and J. A. M. Vermaseren, Nucl. Phys. B 573 (2000) 853 [hep-ph/9912355].

[210] S. Moch, J. A. M. Vermaseren and A. Vogt, Nucl. Phys. B 688 (2004) 101 [hep-ph/0403192];

[211] Nucl. Phys. B 691 (2004) 129 [hep-ph/0404111].

[212] I. Bierenbaum, et al., Nucl. Phys. B 803 (2008) 1 [hep-ph/0803.0273];

[213] K. Wegschaider, Computer generated proofs of binomial multi-sum identities, Master's thesis, RISC, Johannes Kepler University Linz (1997).

[214] M. Kauers, Guessing Handbook, Technical Report RISC 09-07 (2009), JKU Linz.

[215] G. Almkvist and D. Zeilberger, J. Symb. Comp. 10 (1990) 571; M. Apagodu and D. Zeilberger, Adv. Appl. Math. (Special Regev issue), 37 (2006) 139.

[216] C. Koutschan, Advanced Applications of the Holonomic Systems Approach, PhD Thesis, Johannes Kepler University Linz (2009).

[217] J. Ablinger, Computer algebra algorithms for special function in particle physics, $\mathrm{PhD}$ thesis, RISC, Johannes Kepler University Linz, May 2012.

[218] J. Blümlein, Comput. Phys. Commun. 159 (2004) 19 [hep-ph/0311046].

[219] S. Weinzierl, Comput. Phys. Commun. 145 (2002) 357 [math-ph/0201011].

[220] J. Vollinga and S. Weinzierl, Comput. Phys. Commun. 167 (2005) 177 [hep-ph/0410259]. 
[221] D. Maitre, Comput. Phys. Commun. 174 (2006) 222 [hep-ph/0507152].

[222] S. Moch and P. Uwer, Comput. Phys. Commun. 174 (2006) 759 [math-ph/0508008].

[223] S. Buehler and C. Duhr, arXiv:1106.5739 [hep-ph].

[224] J. Blümlein, Comput. Phys. Commun. 133 (2000) 76 [hep-ph/0003100]; in : Proceedings of the Workshop Motives, Quantum Field Theory, and Pseudodifferential Operators, held at the Clay Mathematics Institute, Boston University, June 2-13, 2008, Clay Mathematics Proceedings 12 (2010) pp. 167, Eds. A. Carey, D. Ellwood, S. Paycha, S. Rosenberg, arXiv:0901.0837 [math-ph]; J. Blümlein and S. -O. Moch, Phys. Lett. B 614 (2005) 53 [hep-ph/0503188].

[225] S. Alekhin, J. Blümlein, S. Klein and S. Moch, Phys. Rev. D 81 (2010) 014032 [arXiv:0908.2766 [hep-ph]].

[226] S. Alekhin, J. Blümlein and S. Moch, arXiv:1202.2281 [hep-ph].

[227] HERAPDF, https://www.desy.de/h1 zeus/combined_results/index.php?do= proton_structure.

[228] P. Jimenez-Delgado and E. Reya, Phys. Rev. D 79 (2009) 074023 [arXiv:0810.4274 [hep-ph]].

[229] A. D. Martin, W. J. Stirling, R. S. Thorne and G. Watt, Eur. Phys. J. C 63 (2009) 189 [arXiv:0901.0002 [hep-ph]].

[230] R. D. Ball, V. Bertone, L. Del Debbio, S. Forte, A. Guffanti, J. I. Latorre, S. Lionetti and J. Rojo et al., Phys. Lett. B 707 (2012) 66 [arXiv:1110.2483 [hep-ph]].

[231] F. D. Aaron et al. [H1 and ZEUS Collaboration], JHEP 1001 (2010) 109 [arXiv:0911.0884 [hep-ex]].

[232] J. Blümlein, H. Böttcher and A. Guffanti, Nucl. Phys. B 774 (2007) 182 [hep-ph/0607200].

[233] J. Blümlein, H. Böttcher and A. Guffanti, Nucl. Phys. Proc. Suppl. 135 (2004) 152 [hep-ph/0407089].

[234] J. Blümlein and H. Böttcher, in preparation.

[235] M. Glück, E. Reya and C. Schuck, Nucl. Phys. B 754 (2006) 178 [hep-ph/0604116].

[236] A. D. Martin, W. J. Stirling, R. S. Thorne and G. Watt, Eur. Phys. J. C 64 (2009) 653 [arXiv:0905.3531 [hep-ph]].

[237] P. Nadolsky, News from CTEQ-TEA PDF analysis, July 2011, http: / / indico.desy.de/conferenceDisplay.py?confId=4211 (unpublished).

[238] T. Gehrmann, M. Jaquier and G. Luisoni, Eur. Phys. J. C 67 (2010) 57 [arXiv:0911.2422 [hep-ph]].

[239] R. Abbate, M. Fickinger, A. H. Hoang, V. Mateu and I. W. Stewart, Phys. Rev. D 83 (2011) 074021 [arXiv:1006.3080 [hep-ph]].

[240] G. Dissertori, A. Gehrmann-De Ridder, T. Gehrmann, E. W. N. Glover, G. Heinrich and H. Stenzel, Phys. Rev. Lett. 104 (2010) 072002 [arXiv:0910.4283 [hep-ph]].

[241] P. A. Baikov, K. G. Chetyrkin and J. H. Kühn, Phys. Rev. Lett. 101 (2008) 012002 [arXiv:0801.1821 [hep-ph]].

[242] M. Beneke and M. Jamin, JHEP 0809 (2008) 044 [arXiv:0806.3156 [hep-ph]].

[243] S. Aoki et al. [PACS-CS Collaboration], JHEP 0910 (2009) 053 [arXiv:0906.3906 [hep-lat]].

[244] C. McNeile, C. T. H. Davies, E. Follana, K. Hornbostel and G. P. Lepage, Phys. Rev. D 82 (2010) 034512 [arXiv:1004.4285 [hep-lat]]. 
[245] B. Blossier, P. .Boucaud, M. Brinet, F. De Soto, X. Du, V. Morenas, O. Pene and K. Petrov et al., arXiv:1201.5770 [hep-ph].

[246] S. Bethke, Eur. Phys. J. C 64 (2009) 689 [arXiv:0908.1135 [hep-ph]].

[247] S. Alekhin, J. Blümlein and S. -O. Moch, arXiv:1105.5349 [hep-ph].

[248] S. Alekhin, J. Blümlein, P. Jimenez-Delgado, S. Moch and E. Reya, Phys. Lett. B 697 (2011) 127 [arXiv:1011.6259 [hep-ph]].

[249] G. Aad et al. [ATLAS Collaboration], Phys. Rev. D 85 (2012) 072004 [arXiv:1109.5141 [hep-ex]].

[250] S. Chatrchyan et al. [CMS Collaboration], JHEP 1110 (2011) 132 [arXiv:1107.4789 [hep-ex]].

[251] R. Aaij et al. [LHCb Collaboration], arXiv:1204.1620 [hep-ex].

[252] R. D. Ball, L. Del Debbio, S. Forte, A. Guffanti, J. I. Latorre, J. Rojo and M. Ubiali, Nucl. Phys. B 838 (2010) 136 [arXiv:1002.4407 [hep-ph]].

[253] P. M. Nadolsky, H. -L. Lai, Q. -H. Cao, J. Huston, J. Pumplin, D. Stump, W. -K. Tung and C. -P. Yuan, Phys. Rev. D 78 (2008) 013004 [arXiv:0802.0007 [hep-ph]].

[254] K. Rabbertz, CMS NOTE 2011/004, http: / / cdsweb. cern. ch/collection/CMS2 onotes?ln=de\&as=1.

[255] R. D. Ball, V. Bertone, F. Cerutti, L. Del Debbio, S. Forte, A. Guffanti, J. I. Latorre and J. Rojo et al., Nucl. Phys. B 849 (2011) 296 [arXiv:1101.1300 [hep-ph]].

[256] R. Barate et al. [LEP Working Group for Higgs boson searches and ALEPH and DELPHI and L3 and OPAL Collaborations], Phys. Lett. B 565 (2003) 61 [hep-ex/0306033].

[257] G. Aad et al. [ATLAS Collaboration], arXiv:1202.1408 [hep-ex].

[258] S. Chatrchyan et al. [CMS Collaboration], arXiv:1202.1488 [hep-ex].

[259] H. -L. Lai, M. Guzzi, J. Huston, Z. Li, P. M. Nadolsky, J. Pumplin and C. -P. Yuan, Phys. Rev. D 82 (2010) 074024 [arXiv:1007.2241 [hep-ph]].

[260] W. Beenakker, S. Brensing, M. Kramer, A. Kulesza, E. Laenen and I. Niessen, arXiv:1112.5057 [hep-ph].

[261] K. Sridhar, these Proceedings.

[262] S. K. Majhi, P. Mathews and V. Ravindran, arXiv:1201.1106 [hep-ph].

[263] M. C. Kumar, P. Mathews, V. Ravindran and S. Seth, arXiv:1201.1543 [hep-ph].

[264] A. Tripathi, these Proceedings;

N. Agarwal, V. Ravindran, V. K. Tiwari and A. Tripathi, Phys. Lett. B 690 (2010) 390

[arXiv:1003.5445 [hep-ph]]. 